\title{
Paramodulation with well-founded orderings
}

\author{
Miquel Bofill ${ }^{1}$ and Albert Rubio ${ }^{2}$ \\ 1 Universitat de Girona, Dept. IMA, Girona, Spain \\ mbofill@ima.udg.edu \\ 2 Universitat Politècnica de Catalunya, Dept. LSI, Barcelona, Spain \\ rubio@lsi.upc.edu
}

\begin{abstract}
For many years, all existing completeness results for KnuthBendix completion and ordered paramodulation required the term ordering $\succ$ to be well-founded, monotonic and total(izable) on ground terms. Then, it was shown that well-foundedness and the subterm property were enough for ensuring completeness of ordered paramodulation.

Here we show that the subterm property is not necessary either. By using a new restricted form of rewriting, we obtain a completeness proof of ordered paramodulation for Horn clauses with equality, where wellfoundedness of the ordering suffices. Apart from the theoretical significance of this result, some potential applications motivating the interest of dropping the subterm property are given.

The proof of the results included in this paper, being still technical in some parts, is pretty much shorter and easier to read than the one we have in the preliminary version of this work presented at the CADE 2002 conference [8].
\end{abstract}

Key words: term rewriting, equational reasoning, theorem proving, paramodulation, Knuth-Bendix completion

\section{Introduction}

Equations are ubiquitous in mathematics, logic, and many areas of computer science. Knuth-Bendix-like completion techniques and their extensions to ordered paramodulation for first-order clauses are among the most successful methods for automated deduction with equality $[4,17]$. At the present time most, if not all, state-of-the-art theorem provers for first-order logic with equality are based on variations of the ordered paramodulation inference rule.

These completion and deduction procedures are parameterized by an ordering on terms. For many years, all known completeness results for KnuthBendix completion and ordered paramodulation required the term ordering $\succ$ to be well-founded, monotonic and total (or extendable to a total ordering) on ground terms $[11,2,1,3]$. Under these strong requirements, powerful abstract redundancy notions (covering many practical redundancy elimination techniques such as tautology deletion, subsumption and demodulation) can be applied without losing completeness. However, under certain circumstances, imposing these strong properties on the ordering can be too restrictive, and hence weakening 
them has been a well-known research challenge. For instance, frequently it is not clear what is a good ordering for a particular problem. The following is an interesting example for that. It shows that sometimes Knuth-Bendix completion terminates only if an unusual orientation is chosen for certain equations:

Example 1. ${ }^{3}$ Consider the closure under standard Knuth-Bendix completion of the following two equations:

$$
\begin{aligned}
h(x) & \rightarrow f g(x) \\
h f g(x) & \rightarrow f f g(x)
\end{aligned}
$$

Between these two rules there is a single critical pair since $h f g(z)$ can be rewritten into both $f f g(z)$ and $f g f g(z)$. Therefore in a Knuth-Bendix completion the equation

$$
f f g(z) \simeq f g f g(z)
$$

should be added. If we work with a well-founded and monotonic total ordering then the equation is necessarily oriented into

$$
f g f g(z) \rightarrow f f g(z)
$$

(otherwise we will contradict either well-foundedness or monotonicity or totality on ground terms - see commentaries below-). Unfortunately, with this orientation standard Knuth-Bendix completion would generate an infinite set of rules:

$$
f g f^{n} g(x) \rightarrow f^{n+1} g(x) \quad \text { with } n \geq 1 .
$$

However, if we take the opposite orientation, i.e.,

$$
f f g(x) \rightarrow f g f g(x)
$$

the system is already closed, i.e., standard Knuth-Bendix completion would generate only this additional rule.

It is important to remark that with this orientation, i.e., with $f f g(x) \succ$ $f g f g(x)$, the ordering $\succ$ cannot be extended to a total one, since $f g(x)$ and $g f g(x)$ must be incomparable in any well-founded and monotonic extension of $\succ$ :

- if $f g(x) \succ g f g(x)$ then it contradicts the subterm relation and then by monotonicity we can obtain an infinite decreasing sequence $f g(x) \succ g f g(x) \succ$ $g g f g(x) \succ \ldots$

- if $g f g(x) \succ f g(x)$ then by monotonicity we have $f g f g(x) \succ f f g(x)$ leading to reflexivity and hence to the existence of an infinite decreasing sequence.

Notice that, even if we compute inferences with lefthand sides into righthand sides (in the sense of ordered paramodulation) the system would be closed after adding $h f g(x) \rightarrow f g f g(x)$ to the set.

\footnotetext{
${ }^{3}$ We thank Christopher Lynch for providing us this example.
} 
Another typical situation is in deduction modulo built-in equational theories $E$. There, apart from replacing unification by $E$-unification, the ordering needs to be total up to $E$-equal ground terms and $E$-compatible as well as wellfounded and monotonic (see e.g. [17]). Unfortunately having all these properties can be a too strong requirement. For example, the existence of such an ordering for the case where $E$ consists of associativity and commutativity (AC) properties for some symbols remained open for a long time. ${ }^{4}$ And, even worse, for many theories $E$ such orderings cannot exist. For example, when $E$ contains an idempotency axiom $f(x, x) \simeq x$, then, if $s \succ t$, by monotonicity one should have $f(s, s) \succ f(s, t)$, which by $E$-compatibility implies $s \succ f(s, t)$ and, hence, well-foundedness is lost.

The first results on ordered paramodulation and Knuth-Bendix completion with weaker orderings were given in $[6,7]$. There, the monotonicity requirement was dropped and well-foundedness and the subterm property were shown to be sufficient for completeness. Note that any such ordering can be totalized without losing these two properties. After this, the fundamental question arises whether more requirements can be dropped.

In this paper we prove that, for the case of Horn clauses with equality, the subterm property is not necessary either, that is, equations may be ordered from subterms to superterms such as in $a \rightarrow f(a)$, and ordered paramodulation remains refutation complete. The only requirement left for the ordering is being well-founded (note that $a \rightarrow f(a)$ can be oriented in the well-founded ordering induced by $f^{m}(a) \succ f^{n}(a) \succ a \succ f(a)$ with $\left.m>n>1\right)$.

This is a new important step in the theory of paramodulation, which shows the power of ordered paramodulation regardless of the properties of the ordering that is used, and leaves as the last question whether even well-foundedness is necessary.

Apart from its theoretical value, this result has its most significant potential application in deduction modulo built-in equational theories $E$, since the requirement of a (only) well-founded $E$-compatible ordering does not exclude any theory. Note that, like any denumerable set, the set of $E$-congruence classes admits a well-founded ordering and, hence, there is a well-founded $E$ compatible ordering for any theory $E$. Therefore, if our results are extended to deduction modulo $E$, the only remaining condition to obtain refutation complete $E$-paramodulation calculi relates to the need of computing complete sets of $E$ unifiers in the paramodulation steps. Moreover, we believe that our results can be shown to be compatible with basic strategies $[5,15]$. Then, in fact, as shown in $[19,16]$, if basic strategies are applied, only decidability of the $E$-unification problems is necessary.

Another (less obvious) potential application of our results may be in goaloriented deduction since, in some cases, a goal-oriented (ordered) paramodulation proof can only be obtained if the ordering contradicts the subterm property. Let us illustrate this with a simple example:

\footnotetext{
${ }^{4}$ Once it was found [13,18], it immediately led to a number of follow-up results, such as the decidability of the ground AC-word and -unification problems [12,14].
} 
Example 2. Consider the set of equations

$$
\begin{aligned}
y^{\log _{y} x} & \simeq x \\
\log _{y} y^{x} & \simeq x \\
x^{y+z} & \simeq x^{y} \cdot x^{z}
\end{aligned}
$$

and the goal

$$
\log _{a} b+\log _{a} c \simeq \log _{a}(b \cdot c) .
$$

With the following well-founded ordering

$$
\begin{aligned}
y^{\log _{y} x} & \succ x & & \\
x & \succ \log _{y} y^{x} & & \text { if } x \text { is not headed by log } \\
\log _{y} y^{x} & \succ x & & \text { if } x \text { is headed by log } \\
x^{y+z} & \succ x^{y} \cdot x^{z} & &
\end{aligned}
$$

which contradicts the subterm property, we have a goal-oriented proof by ordered paramodulation (note that some variable renaming have been applied):

$$
\begin{aligned}
& x \simeq \log _{y} y^{x} \quad \log _{a} b+\log _{a} c \not \log _{a}(b \cdot c) \\
& x_{1}^{y_{1}+z_{1}} \simeq x_{1}^{y_{1}} \cdot x_{1}^{z_{1}} \quad \log _{y} y^{\log _{a} b+\log _{a} c} \neq \log _{a}(b \cdot c) \\
& y_{1}^{\log _{y_{1} x_{1}}} \simeq x_{1} \quad \log _{y}\left(y^{\log _{a} b} \cdot y^{\log _{a} c}\right) \not \log _{a}(b \cdot c) \\
& y_{1}^{\log _{y_{1} x_{1}}} \simeq x_{1} \quad \log _{a}\left(b \cdot a^{\log _{a} c} \nsucceq \log _{a}(b \cdot c)\right. \\
& \log _{a}(b \cdot c) \not \log _{a}(b \cdot c)
\end{aligned}
$$

Note that there is no such fully goal-oriented proof with respect to a wellfounded ordering including the subterm relation, because no paramodulation step can then be applied on the goal: for the first two equations, due to the subterm property, only the term on the left can be used and it does not unify with any subterm of the goal; for the last equation neither side overlaps with the goal.

This simple example illustrates that certain expansions of terms, which are possible only if the ordering contradicts the subterm property, can help in obtaining goal-oriented (ordered) paramodulation proofs. Observe, however, that 
the unusual orientation $x \rightarrow \log _{y} y^{x}$ is only taken if $x$ is not headed by log. Therefore, we are not suggesting that it is reasonable to have rules which always have a variable as lefthand side, but having the variable as lefthand side only for some substitutions. Roughly speaking, this approach can be seen as a kind of definition unfolding. The idea is that we can apply such kind of expansion only finitely many times since, although the ordering can contradict the subterm property, it must be well-founded.

\subsection{About the proof technique}

Our proof follows the lines of Bachmair and Ganzinger's model generation proof method of [3] and its variation used in [7] for the non-monotonic case. In this method for proving completeness of ordered paramodulation calculi, a (possibly infinite) ground rewrite system is generated from a closed (or saturated, i.e., closed up to redundancy) set of clauses. This rewrite system $R$ defines an equality Herbrand interpretation $\stackrel{*}{\leftrightarrow}_{R}$, that is shown to be a model of the set of clauses whenever the empty clause is not contained in it (hence the name of "model generation"). The rules in the rewrite system are oriented by the ordering $\succ$ that parameterizes the ordered paramodulation inference rule, and confluence and termination are crucial properties for the completeness proof. In the original proof of [3], confluence is ensured by the fact that the rewrite system is non-overlapping, while termination is due to the fact that $\succ$ is a well-founded and monotonic ordering. In the variation used in [7], confluence is also ensured by the fact that the rewrite system is non-overlapping, but termination is due to the fact the righthand sides are irreducible. In our proof, the rewrite system is neither non-overlapping nor terminating (note that rules of the form $a \rightarrow f(a)$ necessarily cause non-termination of standard rewriting).

To avoid non-termination and keep the simplicity of the original proof, we define a new restricted form of rewriting, called blocked rewriting (B-rewriting for short) which, roughly, blocks the introduced righthand side for future rewriting steps. More precisely, when a rewrite step takes place at some position $p$, then all positions below $p$ - that is, $p$ and all positions strictly below $p$ - are marked as blocked, and further rewrite steps at blocked positions are disallowed.

In order to express these blockings we use abstracted terms, which have variables in the place of blocked subterms. Blocked subterms are stored in a substitution. More precisely, an abstracted term $t \cdot \gamma$ is a term split into two parts: the abstraction (or term) part $t$ and the substitution part $\gamma$, such that $t \gamma$ is a ground term. Variable positions in the term part $t$ correspond to positions at which a rewrite step has taken place, while the substitution part $\gamma$ stores the righthand sides that have been introduced at these positions. That is, we write $t \cdot \gamma \curvearrowright t[x]_{p} \cdot(\gamma \cup\{x \mapsto r\})$, where $x$ is a fresh variable, if $\left.t \gamma\right|_{p}$ is $l, l \rightarrow r$ is a (ground) rewrite rule, and $p$ is a non-variable position of $t$. Note that this relation is well-founded, since $p$ is required to be a non-variable position of $t$ and at every step the number of non-variable symbols of the term part $t$ strictly decreases. Figure 1 shows a rewrite sequence by $B$-rewriting. 


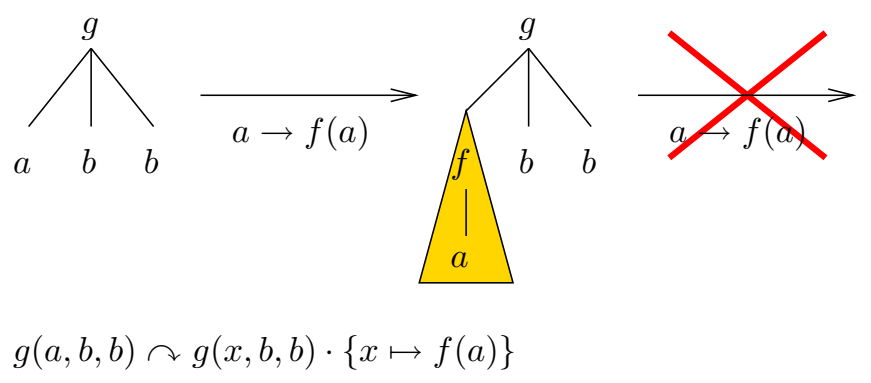

Figure 1. Illustration of $B$-rewriting on abstracted terms.

Abstracted terms correspond, at ground level, to the closures defined for clauses in [5]. However, here they are used only at the completeness proof level, i.e., no blockings appear in actual equations.

As said before, for proving refutation completeness using the model generation technique, another crucial property of the generated rewrite system is confluence, which in all previous results follows trivially from the fact that, by construction, the rewrite system is non-overlapping. Unfortunately, here a nonoverlapping rewrite system cannot be built, and thus finding an appropriate rewrite system with some other properties is necessary. In fact, most of this paper is devoted to the proof of confluence of the generated rewrite system $R$. Once confluence is proved, we can easily show that there exists a $B$-rewrite proof with $R$ for every logical consequence of the set of equations from which $R$ has been generated. Finally, this is used to prove refutation completeness of ordered paramodulation for first-order Horn clauses with eager selection of negative literals. The following example illustrates some of these aspects:

Example 3. Consider a set of equations $E$ containing only

$$
f(a) \simeq a
$$

and an ordering including $a \succ f(a)$ and $f^{k}(a) \succ f^{j}(a)$ for $k \geq 2$ and $k>j \geq 0$. In this ordering, we have $f^{n}(a) \succ a \succ f(a)$ with $n>1$ and, thus, the minimal term is $f(a)$.

If we close $E$ with respect to the ordered paramodulation rule (parameterized by $\succ$ ), we obtain a set of equations of the form

$$
f^{k}(a) \simeq f^{j}(a) \text { for all } k>j \geq 0
$$

which are oriented as

$$
\begin{aligned}
a & \rightarrow f(a) \\
f^{k}(a) & \rightarrow f^{j}(a) \quad \text { for all } k \geq 2 \text { and } k>j \geq 0 .
\end{aligned}
$$


Then the following set $R$ containing the two rules

$$
\begin{aligned}
a & \rightarrow f(a) \\
f(f(a)) & \rightarrow f(a)
\end{aligned}
$$

although being overlapping and non-terminating for standard rewriting, gives us $B$-rewrite proofs for all equations in the closure. In particular, all $f^{k}(a) \simeq f^{j}(a)$ can be proved by joining both sides into the same term $x \cdot\{x \mapsto f(a)\}$ (see Figure 2).

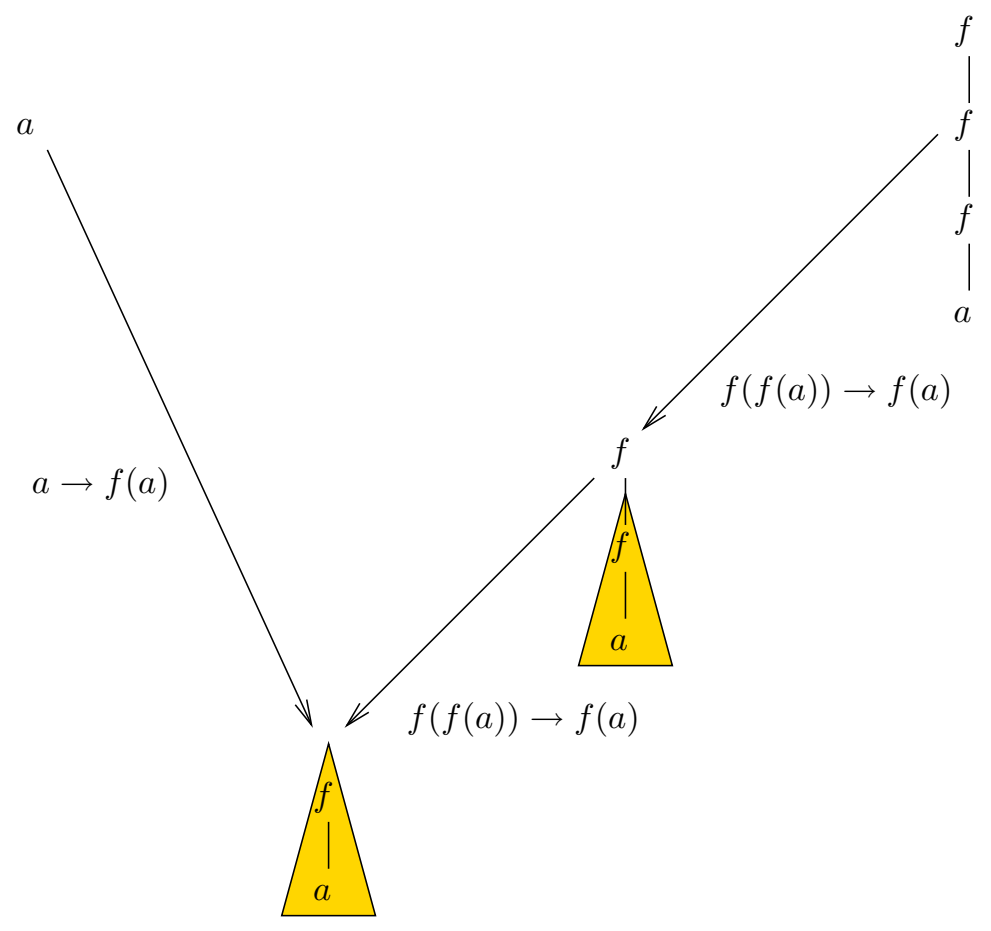

Figure 2. B-rewrite proof from Example 3.

Being still technical in some parts, the new version of the proof included in this paper is pretty much shorter and easier to read than the one we have in the preliminary version of this work presented at the CADE 2002 conference [8].

The paper is structured as follows. In Section 2 we provide the basic definitions. In Section 3 we introduce $B$-rewriting. Section 4 is devoted to the equational case. There, we present the original part of our proof technique, and we show that the limit rewrite system that defines the model gives us $B$-rewrite proofs for all logical consequences. In Section 5, using the results obtained for the 
equational case, we prove refutation completeness of (positive) ordered paramodulation with well-founded orderings for the case of Horn clauses with equality. Section 6 contains the conclusions and possible future developments.

\section{Basic notions}

We use the standard definitions and notation of $[10,17]$.

$T(\mathcal{F}, \mathcal{X})(T(\mathcal{F}))$ is the set of (ground) terms over a set of symbols $\mathcal{F}$ and a denumerable set of variables $\mathcal{X}$ (over $\mathcal{F}$ ).

A position in a term is a sequence of positive integers. Given a term $t$, the subterm of $t$ at position $p$ is denoted by $\left.t\right|_{p}$ : we have $\left.t\right|_{\lambda}=t$ (where $\lambda$ denotes the empty sequence) and $\left.f\left(t_{1}, \ldots, t_{n}\right)\right|_{i . p}=\left.t_{i}\right|_{p}$ if $1 \leq i \leq n$ (and is undefined if $i>n)$. If $\left.s\right|_{p}$ is $t$ and $\left.t\right|_{q}$ is $u$ then we denote by $p \cdot q$ the position of $u$ in $s$, i.e., $\left.s\right|_{p \cdot q}$. The result of replacing $\left.t\right|_{p}$ by $s$ in $t$ is denoted by $t[s]_{p}$. A context $t[]_{p}$ is a term $t$ with a hole (i.e., a lambda-bound variable) at a distinguished position $p$. Then, given a context $t[]_{p}$ and a term $s, t[s]_{p}$ is a term. To avoid confusion, syntactic equality of two terms $s$ and $t$ is denoted by $s \equiv t$. We use $=$ in the metalanguage.

We say that a term $s$ (resp. a function symbol $f$ ) occurs at position $p$ in a term $t$ if $\left.t\right|_{p} \equiv s$ (resp. $\left.t\right|_{p}$ is rooted by $f$ ). By $\operatorname{Var}(t)$ we denote the set of all variables occurring in a term $t$. We write $t \unrhd s$ to indicate that a term $s$ is a subterm of $t$, that is, $s$ occurs in $t$ at some position $p$. If $p \neq \lambda$, we write $t \triangleright s$ and we say that $s$ is a proper subterm of $t$. We speak of position $p$ as being (strictly) above $q$ if $p$ is a (proper) prefix of $q$. At the same time, we speak of $q$ as being (strictly) below $p$. Note that, since a position $p$ is a prefix of itself, $p$ is at the same time above and below $p$. However, $p$ is neither strictly above nor strictly below $p$.

Let $t$ be a term $f\left(t_{1}, \ldots, t_{n}\right)$ in $T(\mathcal{F}, \mathcal{X})$ with $n \geq 0$. Then the size of $t$, denoted by $|t|$, is defined as $\left|t_{1}\right|+\cdots+\left|t_{n}\right|+1$. Note that constants and variables have size 1 ( since $n=0$ ). The size of a term $t$ without counting the variables is denoted by $|t|_{v}$.

We write substitutions as sets of pairs of the form $x \mapsto t$, where $x$ is a variable and $t$ is a term. The domain of a substitution $\sigma$ is denoted by $\operatorname{Dom}(\sigma)$, and its range is denoted by $\operatorname{Ran}(\sigma)$. We use the postfix notation $t \sigma$ for the image of a term $t$ under a substitution $\sigma$, that is, $t \sigma$ denotes $t$ with all variables replaced by terms as specified by $\sigma$. The image of an equation, rewrite rule, or formula under a substitution is defined similarly.

An equation is a multiset of terms $\{s, t\}$, denoted $s \simeq t$ or, equivalently, $t \simeq s$. By $\operatorname{Var}(s \simeq t)$ we denote $\operatorname{Var}(s) \cup \operatorname{Var}(t)$, and by $\operatorname{gnd}(s \simeq t)$ we denote the set of ground instances of $s \simeq t$. When talking about a ground instance $s \simeq t$ of an equation $s^{\prime} \simeq t^{\prime}$ under a substitution $\sigma$, we will assume that $s^{\prime} \sigma \equiv s$ and $t^{\prime} \sigma \equiv t$ in spite of symmetry of equations. Given a set of equations $E$, by $\operatorname{gnd}(E)$ we denote the set of ground instances of all equations in $E$. Similar terminology applies to rewrite rules and formulae in general. 
A first-order equality clause is a pair of finite multisets of equations $\Gamma$ (the antecedent) and $\Delta$ (the succedent), denoted by $\Gamma \rightarrow \Delta$. Clauses $e_{1}, \ldots, e_{n} \rightarrow$ $e_{1}^{\prime}, \ldots, e_{m}^{\prime}$ correspond to the equivalent (quantifier free) disjunction of equations and negated equations $\neg e_{1} \vee \ldots \vee \neg e_{n} \vee e_{1}^{\prime} \vee \ldots \vee e_{m}^{\prime}$. Hence, given a clause $\Gamma \rightarrow \Delta$, equations in $\Gamma$ are called negative equations and equations in $\Delta$ are called positive equations. We also speak of negative and positive literals, though we assume that equality is the only predicate.

If there is no restriction on the number of positive and negative literals, we speak of general clauses. A Horn clause is a clause with at most one positive literal. The empty clause $\square$ is a clause where both $\Gamma$ and $\Delta$ are empty. A positive (resp. negative) clause is a clause $\Gamma \rightarrow \Delta$ where $\Gamma$ (resp. $\Delta$ ) is empty, and a unit clause is a clause with exactly one literal.

If $\rightarrow$ is a binary relation, then $\leftarrow$ is its inverse, $\leftrightarrow$ is its symmetric closure, $\stackrel{+}{\rightarrow}$ is its transitive closure and $\stackrel{*}{\rightarrow}$ is its reflexive-transitive closure. We write $s \stackrel{\bullet}{\rightarrow} t$ if $s \stackrel{*}{\rightarrow} t$ and there is no $t^{\prime}$ such that $t \rightarrow t^{\prime}$. Then $t$ is called irreducible and a normal form of $s$ (w.r.t. $\rightarrow$ ). If a term $s$ has exactly one normal form then it is denoted by $s ! \rightarrow$. The relation $\rightarrow$ is well-founded or terminating if there exists no infinite sequence $s_{1} \rightarrow s_{2} \rightarrow \ldots$ and it is confluent or Church-Rosser if the relation $\leftarrow^{*} \circ \rightarrow^{*}$ is contained in $\rightarrow^{*} \circ \leftarrow^{*}$. It is locally confluent if $\leftarrow \circ \rightarrow \subseteq \rightarrow^{*} \circ \leftarrow^{*}$. By Newman's lemma, terminating locally-confluent relations are confluent. A relation $\rightarrow$ on terms is monotonic if $s \rightarrow t$ implies $u[s]_{p} \rightarrow u[t]_{p}$ for all terms $s$, $t$ and $u$ and positions $p$. An equivalence relation is a reflexive, symmetric and transitive relation. A congruence is a monotonic equivalence relation on terms.

The multiset extension of an equivalence relation $\sim$ is defined as the smallest relation $\sim^{m u l}$ on multisets such that $\emptyset \sim^{m u l} \emptyset$, and $S \cup\{s\} \sim^{m u l} S^{\prime} \cup\{t\}$ if $S \sim^{m u l} S^{\prime}$ and $s \sim t$.

A rewrite rule is an ordered pair of terms $(s, t)$, written $s \rightarrow t$, and a set of rewrite rules $R$ is a term rewrite system (TRS). The rewrite relation with $R$ on $T(\mathcal{F}, \mathcal{X})$, denoted by $\rightarrow_{R}$, is the smallest monotonic relation such that for all rules $l \rightarrow r$ in $R$ and substitutions $\sigma$, it includes $l \sigma \rightarrow_{R} r \sigma$. That is, $s \rightarrow_{R} t$ if and only if $s \equiv u[l \sigma]_{p}$ and $t \equiv u[r \sigma]_{p}$ for some context $u$, position $p$ in $u$, rule $l \rightarrow r$ in $R$ and substitution $\sigma$; then we say that $s$ rewrites into $t$ with $R$, and that $s$ is reducible by $R$. (It is commonly assumed that lefthand sides of rules are not variables, and that all variables occurring in a righthand side also occur in the corresponding lefthand side.) A subterm $\left.s\right|_{p}$ at which a rewrite (with $R$ ) can take place is called a $(R$-) redex. A redex $r$ is innermost for $R$ if it is an $R$-redex but no proper subterms of $r$ are $R$-redexes. A derivation $s_{1} \rightarrow_{R} s_{2} \rightarrow_{R} \cdots$ for a rewrite system $R$ is called a rewrite sequence with $R$. It is called innermost if all rewrite steps take place at innermost redexes. A rewrite system $R$ is called terminating, confluent, etc. if $\rightarrow_{R}$ is. It is called convergent if it is confluent and terminating; then every term $t$ has a unique normal form w.r.t. $\rightarrow_{R}$, denoted by $n f_{R}(t)$, and $s \simeq t$ is a logical consequence of $R$ (where $R$ is seen as a set of equations) if and only if $n f_{R}(s)$ and $n f_{R}(t)$ are the same term. It is called overlapping if there are two rules $l \rightarrow r$ and $s \rightarrow t$ such that $l$ and some non-variable subterm $\left.s\right|_{p}$ of $s$ have a common instance, i.e., they unify. 
Let $R$ be a ground TRS. Then the congruence $\leftrightarrow_{R}^{*}$ defines an equality Herbrand interpretation, where the only predicate $\simeq$ is interpreted by $s \simeq t$ iff $s \leftrightarrow{ }_{R}^{*} t$. Such an interpretation will be denoted by $R^{*}$. We write $s \simeq t \in R^{*}$ if $s \leftrightarrow_{R}^{*}$ t. $R^{*}$ satisfies (is a model of) a ground clause $\Gamma \rightarrow \Delta$, denoted $R^{*} \models \Gamma \rightarrow \Delta$, if $R^{*} \nsupseteq \Gamma$ or $R^{*} \cap \Delta \neq \emptyset$. The empty clause $\square$ is hence satisfied by no interpretation. $R^{*}$ satisfies a clause $C$ if it satisfies every ground instance of $C$. And $R^{*}$ satisfies a set of clauses $S$, denoted by $R^{*} \models S$, if it satisfies all clauses in $S$.

A quasi-ordering $\succeq$ is a transitive and reflexive binary relation. The subsumption relation, denoted by $\geq$, is a quasi-ordering defined as $s \geq t$ if $s$ is an instance of $t$, i.e., $s \equiv t \sigma$ for some $\sigma$. A (strict partial) ordering $\succ$ is a transitive and irreflexive binary relation. An ordering on $T(\mathcal{F}, \mathcal{X})$ is a reduction ordering if it is well-founded and monotonic, and moreover, it is stable under substitutions: $s \succ t$ implies $s \sigma \succ t \sigma$ for all substitutions $\sigma$. It fulfills the subterm property if $\succ \supseteq \triangleright$, where $\triangleright$ denotes the strict subterm ordering. It is total on $T(\mathcal{F})$ if for all $s$ and $t$ in $T(\mathcal{F})$, either $s \equiv t$ or $s \succ t$ or $t \succ s$. A west-ordering [7] is a well-founded ordering on $T(\mathcal{F})$ that fulfills the subterm property and that is total on $T(\mathcal{F})$ (it is called west after well-founded, subterm and total). Every well-founded ordering can be totalized [20], and hence every well-founded ordering satisfying the subterm property can be extended to a west-ordering. It also holds that every reduction ordering can be extended to a west-ordering [7].

\section{Blocked rewriting}

Here we define a special kind of rewrite relation, called blocked rewriting ( $B$ rewriting for short), which is an essential ingredient for our completeness proof. (See Section 1 for the motivation of this new kind of rewrite relation.)

This relation restricts standard rewriting by mainly forbidding rewrite steps below righthand sides introduced in previous rewrite steps. Roughly speaking, at every rewrite step, the introduced righthand side becomes blocked for further rewrite steps. This makes this relation terminating by definition.

In order to express these blockings, we use abstracted terms, which have variables in the place of the blocked subterms. Blocked subterms are stored in a substitution. This formalism corresponds, at ground level, to the closures defined for clauses in [5]. However, here this is used only at the completeness proof level, i.e., no blockings appear in actual equations.

Definition 1 (Abstracted term). Let $s$ be a term in $T(\mathcal{F}, \mathcal{X})$ and $\gamma$ a ground substitution, such that $\operatorname{Var}(s) \subseteq \operatorname{Dom}(\gamma)$. Then:

1. $s \cdot \gamma$ is called an abstracted term. We may write $s$ instead of $s \cdot \gamma$ whenever $s$ is ground.

2. $s \cdot \gamma$ is said to be an abstraction of a term $t$ if $s \gamma \equiv t$.

3. $s \cdot \gamma$ is said to be an abstraction w.r.t. a set of terms $T$ if for all variables $x$ of $s$, we have that $x \gamma \in T$.

4. Variable positions of $s$ are called abstracted (or blocked) positions of $s \cdot \gamma$. 
5. Two abstracted terms $s \cdot \gamma$ and $u \cdot \delta$ are called equivalent, denoted by $s \cdot \gamma \equiv t \cdot \delta$, if $s$ and thave the same variable positions and $s \gamma \equiv u \delta$.

We refer to an equation between abstracted terms as an abstracted equation, and we may write $(s \simeq t) \cdot \gamma$ for $s \cdot \gamma \simeq t \cdot \gamma$. When talking about an abstraction $(s \simeq t) \cdot \gamma$ of an equation $u \simeq v$, we will assume, in spite of symmetry of equations, that $s \cdot \gamma$ is an abstraction of $u$ and $t \cdot \gamma$ is an abstraction of $v$. A clause consisting of abstracted equations is called an abstracted clause, and it may analogously be denoted by $C \cdot \gamma$, where $C$ is a clause. We may also write e instead of $e \cdot \gamma$ (resp. $C$ instead of $C \cdot \gamma$ ) whenever the equation e (resp. clause $C$ ) is ground. The notion of abstraction w.r.t. $T$ is extended to equations and clauses in the natural way.

Given a rewrite system $R$, by $\operatorname{Left}(R)$ and $\operatorname{Right}(R)$ we denote, respectively, the set of lefthand sides of rules in $R$ and the set of righthand sides of rules in $R$. In what follows we only consider ground rewrite systems, which is the only case we need, but all definitions can easily be extended to deal with non-ground rewrite systems.

Now we define $B$-rewriting as a rewrite relation on abstracted terms:

Definition 2 (B-rewriting). Let $s \cdot \gamma$ and $t \cdot \delta$ be two abstracted terms, $R$ be a ground TRS and $p$ be a position. Then

$$
s \cdot \gamma \curvearrowright_{R}^{p} t \cdot \delta \quad \text { iff }
$$

1. $p$ is a non-variable position of $s$,

(a) there is some rule $\left.s\right|_{p} \gamma \rightarrow r$ in $R$ or

(b) $\left.s\right|_{p} \gamma \equiv r$ for some $r$ in $\operatorname{Right}(R)$, and

2. $t \cdot \delta \equiv s[x]_{p} \cdot(\gamma \cup\{x \mapsto r\})$ for some fresh variable $x$.

We speak of $p$ as being a reducible position of $s \cdot \gamma$ by $\curvearrowright_{R}$. We may often write $s \cdot \gamma \curvearrowright_{R} t \cdot \delta$ when the position $p$ is not relevant or clear from the context.

Note that if $s \cdot \gamma$ is an abstracted term w.r.t. $\operatorname{Right}(R)$ then so is $t \cdot \delta$. Note also that this rewrite relation not only blocks the introduced righthand sides of $R$, but also righthand sides of $R$ that are already present in the ground version of the abstracted term. We refer to these latter rewrite steps as abstraction steps. Their existence is due to technical reasons, related to equivalence of abstracted terms obtained by $B$-rewriting.

Lemma 1. Let $R$ be a ground TRS. Then $\curvearrowright_{R}$ is terminating on abstracted terms.

Proof. At every rewrite step by $\curvearrowright_{R}$, the number of non-variable symbols of the term part strictly decreases. Hence, $\curvearrowright_{R}$ is terminating by definition. 


\section{Paramodulation with equations}

This section is devoted to the pure equational case. In the following, we will assume that $\succ$ is a given (total) well-founded ordering on ground terms. We will also assume that all equations at hand have disjoint variables (if necessary, they can be renamed).

\subsection{The inference system}

Definition $3(\mathcal{E})$. The inference rule $\mathcal{E}$ of (equational) ordered paramodulation with respect to $\succ$ is:

$$
\frac{l \simeq r \quad s \simeq t}{\left(s[r]_{p} \simeq t\right) \theta}
$$

where $\theta=\operatorname{mgu}\left(\left.s\right|_{p}, l\right)$ is the most general unifier of $\left.s\right|_{p}$ and $l, l$ is maximal in its premise (that is, for some ground substitution $\sigma$, it holds that $l \theta \sigma \succ r \theta \sigma$ ), and $\left.s\right|_{p}$ is not a variable, or $l$ and $s$ are both variables.

We will assume that, if $l$ is a variable $x$, then $x$ occurs in $r$ since, otherwise, the theory is trivial. Note that this situation is possible, together with $l \theta \sigma \succ r \theta \sigma$, as the ordering $\succ$ is allowed to contradict the subterm property. The inference with such an equation, when $s$ is a variable $y$, has $(r \simeq t) \theta$ as conclusion, where $\theta$ is just the variable renaming of $y$ by $x$ (or of $x$ by $y$ ). Hence, these potential inferences at variable positions are not especially prolific, as they just compute transitivity, and moreover they are not possible if the ordering fulfills the subterm property.

We must note that the need of paramodulations from variables into variables at top positions has to do with our proof technique. At present, we have not found any counterexample showing incompleteness when paramodulation into variables is completely disallowed.

Now we define a well-founded ordering $\succ_{e}$ on ground equations, which will be used in the inductive definition of the limit TRS that determines the model of a given set of equations.

Definition $4\left(\succ_{e}\right)$. Let $\succ$ be the well-founded ordering used in the ordered paramodulation inference rule $\mathcal{E}$. We define the relation $\succ_{e}$ on ground equations as follows: given two ground equations $s \simeq t$ and $u \simeq v$, we have

$$
s \simeq t \succ_{e} u \simeq v \quad \text { iff }
$$

1. $s \succeq t$,

2. $u \succeq v$, and
(a) $|s|>|u|$, or
(b) $|s|=|u|$ and $s \succ u$.
(c) $s \equiv u$ and $t \succ v$.

It is worth noting that, for our purposes, comparing the big sides $s$ and $u$ with any well-founded ordering including the subterm relation would suffice, and thus cases $(a)$ and $(b)$ are just one possible choice of comparison. 
Lemma 2. $\succ_{e}$ is a well-founded ordering on ground equations.

Proof. The relation $\succ_{e}$ can be seen as the lexicographic combination of (a) a well-founded quasi-ordering on ground equations, comparing the length of the big sides, (b) a well-founded quasi-ordering on ground equations comparing the big sides with a well-founded ordering when the big sides are equal in length, and (c) a well-founded ordering on ground equations, comparing the small sides with a well-founded ordering when the big sides are the same. Hence, $\succ_{e}$ is a well-founded ordering on ground equations.

Observe also that if $\succ$ is total on ground terms, then so is $\succ_{e}$ on ground equations.

\subsection{The rewrite system}

Given a set of equations $E$, now we define a ground TRS $R_{E}$ generated by $E$, which is the key for the refutation completeness proof. As said, this TRS $R_{E}$ will be shown to provide rewrite proofs by $B$-rewriting for all logical consequences of $E$ whenever $E$ is closed under ordered paramodulation.

In order to take into account the fact that $R_{E}$ will be used applying $B$ rewriting, and hence the righthand sides will become blocked after each rewrite step, in the construction of $R_{E}$ we will not require irreducibility at those positions that are below subterms belonging to $\operatorname{Right}\left(R_{E}\right)$. For instance, if $a \rightarrow f(a)$ belongs to $R_{E}$ then in the term $g(f(a))$, since $f(a)$ is in $\operatorname{Right}\left(R_{E}\right)$, the subterm $f(a)$ is not required to be irreducible. The following definition will be used to describe the only set of positions of a given term $t$ that will be required to be irreducible in this sense.

Definition 5 (Strict context). Let $t$ be a ground term and $T$ a set of ground terms. By the strict context of $t$ with respect to $T$, denoted by StrictContext $(t, T)$, we refer to the set of positions $p$ of $t$ such that there is no prefix $p^{\prime}$ of $p$ with $\left.t\right|_{p^{\prime}} \in T$.

Example 4. StrictContext $(f(g(a), h(a)),\{g(a), a\})$ are the non-hole positions of the context $f([], h([]))$, i.e., just the positions $\lambda$ and 2 .

A closely related concept, which will be used later on, is the following.

Definition 6 (Border). Let $t$ be a ground term and $T$ a set of ground terms. We define the border of $t$ w.r.t. $T$ as follows:

$$
\operatorname{Border}(t, T)=\operatorname{Strict} C o n t e x t(t, T \backslash\{t\}) .
$$

Intuitively, Border $(t, T)$ discards only those positions that are below strict subterms of $t$ in $T$. 
Example 5. We have that

$$
\text { StrictContext }(h(f(a), b),\{f(a), h(f(a), b)\})=\emptyset,
$$

whereas

$$
\begin{gathered}
\text { Border }(h(f(a), b),\{f(a), h(f(a), b)\})= \\
\text { StrictContext }(h(f(a), b),\{f(a)\})=\{\lambda, 2\} .
\end{gathered}
$$

Now we can build the TRS $R_{E}$ choosing the appropriate ground instances of equations in $E$, by induction on $\succ_{e}$.

Definition $7\left(R_{E}\right)$. Let $E$ be a set of equations. Then, a ground instance $e$ of the form $\left(s^{\prime} \simeq t^{\prime}\right) \sigma$ of an equation $s^{\prime} \simeq t^{\prime}$ in $E$ generates the rule $s^{\prime} \sigma \rightarrow t^{\prime} \sigma$ in $R_{E}$ if, and only if,

1. $s^{\prime} \sigma \succ t^{\prime} \sigma$,

2. $s^{\prime} \sigma$ is irreducible by $R_{E}^{e}$ at every position in $\operatorname{Strict} \operatorname{Context}\left(s^{\prime} \sigma, \operatorname{Right}\left(R_{E}^{e}\right)\right)$, and

3. $t^{\prime} \sigma$ is irreducible by $R_{E}^{e}$ at every position in StrictContext $\left(t^{\prime} \sigma, \operatorname{Right}\left(R_{E}^{e}\right)\right)$.

where $R_{E}^{e}$ is the set of rules generated by all instances $d$ of equations in $E$ such that $e \succ_{e} d$.

We denote by $R_{E}$ the set of rules generated by all ground instances of $E$.

Example 6. For the closed set of equations $E$ in Example 3 of Section $1, R_{E}$ consists of the following rules:

$$
\begin{aligned}
a & \rightarrow f(a) \\
f(f(a)) & \rightarrow f(a)
\end{aligned}
$$

Using a relation which includes the subterm relation, for comparing the maximal sides of the ground instances of the equations in $E$, is crucial in order to ensure that the lefthand sides of the rules in $R_{E}$ only overlap at positions below subterms in $\operatorname{Right}\left(R_{E}\right)$.

The way we define $R_{E}$ is quite similar to the definition in [3], and especially to the one in [7]. The main difference is that left- and righthand sides of rules can be reducible at positions below subterms in $\operatorname{Right}\left(R_{E}\right)$. Interestingly, if the well-founded ordering $\succ$ used in the ordered paramodulation inference rule is indeed a west ordering (i.e., it fulfills the subterm property as well), then the generated $R_{E}$ coincides with the rewrite system generated in [7].

We associate the following ground TRS $\vec{E}$ to a set of equations $E$.

Definition $8(\vec{E})$. Let $E$ be a set of equations. We define $\vec{E}$ as the ground TRS obtained by orienting w.r.t. $\succ$ the ground instances of all equations in E, i.e.,

$$
\vec{E}=\{s \rightarrow t \mid s \simeq t \in \operatorname{gnd}(E) \wedge s \succ t\} .
$$


Observe that $R_{E} \subseteq \vec{E}$. There is a subset of $\vec{E}$ which is closely related to $R_{E}$, which will play a central role in our proofs: the set of rules $s \rightarrow t$ such that $t \in \operatorname{Right}\left(R_{E}\right)$.

Definition $9\left(R_{E}^{\circ}\right)$. Let $E$ be a set of equations. We define

$$
R_{E}^{\circ}=\left\{s \rightarrow t \mid s \rightarrow t \in \vec{E} \wedge t \in \operatorname{Right}\left(R_{E}\right)\right\} .
$$

Hence we have $R_{E} \subseteq R_{E}^{\circ} \subseteq \vec{E}$. For technical reasons, many of the results are proved for $R_{E}^{\circ}$. Then, the results for $R_{E}$ follow as a corollary.

Example 7. Again, following Example 3, $R_{E}^{\circ}$ consists of the rules

$$
\begin{aligned}
a & \rightarrow f(a) \\
f^{k}(a) & \rightarrow f(a) \quad \text { for all } k \geq 2 .
\end{aligned}
$$

\subsection{Some relevant properties}

The following lemmas state several relevant properties of $R_{E}$ and $R_{E}^{\circ}$, which will be used in the proof of confluence of $R_{E}$ and, in particular, to show the so-called Return Property (see Subsection 4.4). Recall that the generated $R_{E}$ can be overlapping and non-terminating and, hence, confluence does not follow easily.

A crucial property of $R_{E}$ is that if, at some point of its construction, i.e., for some $R_{E}^{e}$, a term $t$ is reducible at $\operatorname{Strict} \operatorname{Context}\left(t, \operatorname{Right}\left(R_{E}^{e}\right)\right)$, then $t$ will not become a righthand side of $R_{E}$.

Lemma 3. Let $E$ be a set of equations and $t$ a ground term. If, for some ground instance $e$ of an equation in $E, t$ is reducible by $R_{E}^{e}$ at some position $p$ in StrictContext $\left(t, \operatorname{Right}\left(R_{E}^{e}\right)\right)$, then $p$ is in StrictContext $\left(t, \operatorname{Right}\left(R_{E}\right)\right)$.

Proof. We proceed by contradiction. Let $l \rightarrow r$ be the rule in $R_{E}^{e}$ that reduces $t$ at position $p$, and suppose that $p \notin \operatorname{Strict} \operatorname{Context}\left(t, \operatorname{Right}\left(R_{E}\right)\right)$. Then necessarily there is some term $u$ in $\operatorname{Right}\left(R_{E}\right) \backslash \operatorname{Right}\left(R_{E}^{e}\right)$ such that $\left.t\right|_{p^{\prime}} \equiv u$ for some prefix $p^{\prime}$ of $p$.

Therefore, there is an equation $d$ in $\operatorname{gnd}(E)$ of the form $s \simeq u$, with $s \succ u$, that generates a rule $s \rightarrow u$ in $R_{E}$. We choose such an equation $d$ to be minimal w.r.t. $\succ_{e}$, i.e., we assume that $d$ is the smallest equation w.r.t. $\succ_{e}$ in $\operatorname{gnd}(E)$ that generates a rule whose righthand side is a superterm of $l$. Therefore, we have that $\left.u\right|_{p^{\prime \prime}} \equiv l$ for some position $p^{\prime \prime}$ in $\operatorname{Strict} \operatorname{Context}\left(u, \operatorname{Right}\left(R_{E}^{d}\right)\right)$. Finally, we have the following:

- If $e \succ_{e} d$ then $s \rightarrow u \in R_{E}^{e}$, contradicting $u \in \operatorname{Right}\left(R_{E}\right) \backslash \operatorname{Right}\left(R_{E}^{e}\right)$.

- If $e \nsucc_{e} d$ then $R_{E}^{e} \subseteq R_{E}^{d}$, and thus $l \rightarrow r \in R_{E}^{d}$. But since, as stated before, we have that $\left.u\right|_{p^{\prime \prime}} \equiv l$ for some position $p^{\prime \prime}$ in $\operatorname{Strict} \operatorname{Context}\left(u, \operatorname{Right}\left(R_{E}^{d}\right)\right)$, then $d$ fails Condition 3 to generate a rule in $R_{E}$, contradicting the assumption. 
Lemma 4. Let $E$ be a set of equations, and t a term in Right $\left(R_{E}\right)$. Then, for every instance e of an equation in $E, t$ is irreducible by $R_{E}^{e}$ at every position in StrictContext $\left(t, \operatorname{Right}\left(R_{E}^{e}\right)\right)$.

Proof. We proceed by contradiction. If, for some ground instance $e$ of an equation in $E, t$ was reducible by $R_{E}^{e}$ at some position $p$ in $\operatorname{Strict} \operatorname{Context}\left(t, \operatorname{Right}\left(R_{E}^{e}\right)\right)$, then, by Lemma 3, $p$ would be in $\operatorname{Strict} \operatorname{Context}\left(t, \operatorname{Right}\left(R_{E}\right)\right)$. But this is not possible if $t$ is a righthand side of $R_{E}$ since, in that case, the set of positions in StrictContext $\left(t, \operatorname{Right}\left(R_{E}\right)\right)$ is empty.

As a consequence, by construction, all rules $s \rightarrow t$ that are not included in $R_{E}$ will be reducible, in one of their sides, at some position which is strictly above all its subterms belonging to $\operatorname{Right}\left(R_{E}^{s \simeq t}\right)$. This happens in particular to the lefthand sides of the rules in $R_{E}^{\circ} \backslash R_{E}$.

Lemma 5. Let $E$ be a set of equations. For every rule $s \rightarrow t$ in $R_{E}^{\circ} \backslash R_{E}$ we have that $s$ is reducible by $R_{E}^{s \simeq t}$ at some position in StrictContext $\left(s, \operatorname{Right}\left(R_{E}^{s \simeq t}\right)\right)$.

Proof. We proceed by contradiction. Let $s \rightarrow t$ be a rule in $R_{E}^{\circ} \backslash R_{E}$, and suppose that $s$ is irreducible by $R_{E}^{s \simeq t}$ at every position in $\operatorname{Strict} \operatorname{Context}\left(s, \operatorname{Right}\left(R_{E}^{s \simeq t}\right)\right)$. Then, from the facts that (i) $s \succ t$, (ii) $s$ is irreducible by $R_{E}^{s \simeq t}$ at every position in StrictContext $\left(s, \operatorname{Right}\left(R_{E}^{s \simeq t}\right)\right)$ and (iii) $s \rightarrow t \notin R_{E}$, we have that $t$ must be reducible by $R_{E}^{s \simeq t}$ at some position $p$ in $\operatorname{Strict} \operatorname{Context}\left(t, \operatorname{Right}\left(R_{E}^{s \simeq t}\right)\right)$. But then, since $t$ is a righthand side of $R_{E}^{\circ}$ and hence of $R_{E}$, this contradicts Lemma 4.

Lemma 6. Let $E$ be a set of equations. For every ground term $t$, we have that $t$ is reducible by $R_{E}$ at Border $\left(t, \operatorname{Right}\left(R_{E}\right)\right)$ if and only if $t$ is reducible by $R_{E}^{\circ}$ at $\operatorname{Border}\left(t, \operatorname{Right}\left(R_{E}\right)\right)$.

Proof. The left-to-right implication holds since $R_{E} \subseteq R_{E}^{\circ}$. For the right-toleft implication, let $l \rightarrow r$ be a rule in $R_{E}^{\circ}$ that reduces $t$ at some position $q$ in $\operatorname{Border}\left(t, \operatorname{Right}\left(R_{E}\right)\right)$. If $l \rightarrow r$ belongs to $R_{E}$ we are done. Otherwise,

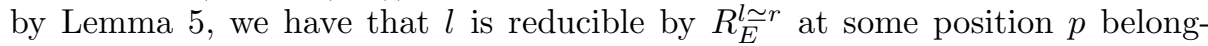
ing to StrictContext $\left(l, \operatorname{Right}\left(R_{E}^{l \sim} r\right)\right)$, i.e., in $\operatorname{Strict} \operatorname{Context}\left(\left.t\right|_{q}, \operatorname{Right}\left(R_{\bar{E}}^{l \sim r}\right)\right)$, and, hence, by Lemma 3, $p$ is in StrictContext $\left(\left.t\right|_{q}, \operatorname{Right}\left(R_{E}\right)\right)$. Thus, $\left.t\right|_{q}$ is reducible by $R_{E}$ at some position $p$ in StrictContext $\left(\left.t\right|_{q}, \operatorname{Right}\left(R_{E}\right)\right)$ and, since $q$ is in $\operatorname{Border}\left(t, \operatorname{Right}\left(R_{E}\right)\right)$, we have $t$ reducible at $q \cdot p$ in $\operatorname{Border}\left(t, \operatorname{Right}\left(R_{E}\right)\right)$ by $R_{E}$.

Another crucial property of $R_{E}$ is that its lefthand sides only overlap below subterms corresponding to righthand sides.

Lemma 7. Let $E$ be a set of equations. For every rule $s \rightarrow t$ in $R_{E}$ we have that $s$ is irreducible by $R_{E} \backslash\{s \rightarrow t\}$ at all positions in StrictContext $\left(s, \operatorname{Right}\left(R_{E}^{s \simeq t}\right)\right)$, and hence at all positions in StrictContext $\left(s, \operatorname{Right}\left(R_{E}\right)\right)$. 
Proof. We proceed by contradiction. Let $s \rightarrow t$ be a rule in $R_{E}$, and suppose that $s$ is reducible by some rule $l \rightarrow r$ in $R_{E} \backslash\{s \rightarrow t\}$ at some position belonging to $\operatorname{StrictContext}\left(s, \operatorname{Right}\left(R_{E}^{s \simeq t}\right)\right)$. Two possibilities have to be considered:

1. $s \triangleright l$. Then we have that $s \simeq t \succ_{e} l \simeq r$, since $s \succ t, l \succ r$, and $|s|>|l|$ (see Definition 4). Therefore, $s$ is reducible by $R_{E}^{s \simeq t}$ at some position in StrictContext $\left(s, \operatorname{Right}\left(R_{E}^{s \simeq t}\right)\right)$, contradicting Condition 2 to generate a rule in $R_{E}$.

2. $s \equiv l$. In this case assume, w.l.o.g., that $s \simeq t \succ_{e} l \simeq r$ (since $s \equiv l$, the other case is analogous). Then we can conclude as in the previous case.

Finally, since $R_{E}^{s \simeq t}$ is included in $R_{E}$, we have $\operatorname{Strict} \operatorname{Context}\left(s, \operatorname{Right}\left(R_{E}\right)\right) \subseteq$ StrictContext $\left(s, \operatorname{Right}\left(R_{E}^{s \simeq t}\right)\right)$, which implies that $s$ is irreducible at all positions in $\operatorname{Strict}$ Context $\left(s, \operatorname{Right}\left(R_{E}\right)\right)$.

This property generalizes the notion in [7] of irreducibility for the rules of the ground TRS that defines the model. Here, irreducibility amounts to irreducibility strictly above subterms corresponding to righthand sides. Therefore, in particular, if the righthand sides of the rules in $R_{E}$ are irreducible by $R_{E}$, then the lefthand sides are irreducible as well, and both notions of irreducibility coincide.

The following lemma is a consequence of the previous one.

Lemma 8. Let $E$ be a set of equations, and $s \rightarrow t$ be a rule in $R_{E}$. If $s$ is reducible, at some position $p$, by a rule $l \rightarrow r$ in $R_{E}^{\circ}$ such that $s \simeq t \succ_{e} l \simeq r$, then there is some position $p^{\prime}$ above $p$ in $s$ such that $\left.s\right|_{p^{\prime}} \in \operatorname{Right}\left(R_{E}\right)$.

Proof. We proceed by contradiction. We have that, for every position $p$ of $s$, if there is no position $p^{\prime}$ above $p$ such that $\left.s\right|_{p^{\prime}} \in \operatorname{Right}\left(R_{E}\right)$, then $p$ is a position in StrictContext $\left(s, \operatorname{Right}\left(R_{E}\right)\right)$. Now suppose that $s$ is reducible, at such position $p$, by some rule $l \rightarrow r$ in $R_{E}^{\circ}$ such that $s \simeq t \succ_{e} l \simeq r$, and recall that $R_{E} \subseteq R_{E}^{\circ}$.

If $l \rightarrow r$ belongs to $R_{E}$ then, since $s \simeq t \succ_{e} l \simeq r, s$ would be reducible by $R_{E} \backslash\{s \rightarrow t\}$ at a position in $\operatorname{StrictContext}\left(s, \operatorname{Right}\left(R_{E}\right)\right)$, contradicting Lemma 7 .

If $l \rightarrow r$ belongs to $R_{E}^{\circ} \backslash R_{E}$ then, by Lemma $5, l$ would be reducible by $R_{E}^{l \sim r}$ at some position $q$ in StrictContext $\left(l, \operatorname{Right}\left(R_{E}^{l \sim r}\right)\right)$ and, by Lemma 3, in $\operatorname{Strict} \operatorname{Context}\left(l, \operatorname{Right}\left(R_{E}\right)\right)$. Therefore, since $\left.s\right|_{p} \equiv l$ and $p$ is a position in $\operatorname{Strict} \operatorname{Context}\left(s, \operatorname{Right}\left(R_{E}\right)\right), p \cdot q$ would be in $\operatorname{Strict} \operatorname{Context}\left(s, \operatorname{Right}\left(R_{E}\right)\right)$. Moreover, since $s \simeq t \succ_{e} l \simeq r$, the rule in $R_{E}^{l \simeq r}$ reducing $l$ at position $q$ would belong to $R_{E} \backslash\{s \rightarrow t\}$. From these facts, a contradiction with Lemma 7 follows as before.

The following two lemmas roughly establish what kind of inferences are necessary and thus which equations can be assumed to belong to $E$ whenever it is closed under $\mathcal{E}$. 
Lemma 9. Let $E$ be a set of equations that is closed under $\mathcal{E}, s^{\prime} \simeq t^{\prime}$ an equation in $E, \sigma$ a ground substitution, and $t \cdot \gamma$ an abstraction of $t^{\prime} \sigma$. If

$$
t \cdot \gamma \curvearrowright_{\vec{E}}^{p_{1}} t_{1} \cdot \gamma \cdots \curvearrowright_{\vec{E}}^{p_{n}} t_{n} \cdot \gamma
$$

where, for all $i$ in $\{1, \ldots, n\}, p_{i}$ is not below any variable position of $t^{\prime}$ and $t_{i} \gamma \not \equiv s^{\prime} \sigma$, then there is an equation $s^{\prime \prime} \simeq t^{\prime \prime}$ in $E$, where $s^{\prime \prime}$ is subsumed by $s^{\prime}$ and $t^{\prime \prime}[]_{p_{1}} \cdots[]_{p_{n}}$ is subsumed by $t^{\prime}[]_{p_{1}} \cdots[]_{p_{n}}$, that has an instance of the form $s^{\prime} \sigma \simeq t_{n} \gamma$.

Proof. We proceed by induction on the number of steps of the rewrite sequence. If there is no step, i.e., if $n=0$, then, taking $s^{\prime} \simeq t^{\prime}$ for $s^{\prime \prime} \simeq t^{\prime \prime}$, we are done by the assumptions. Otherwise, we have that $t \cdot \gamma \curvearrowright \frac{p_{1}}{\vec{E}} t_{1} \cdot \gamma$ for some position $p_{1}$ and some abstracted term $t_{1} \cdot \gamma$. If this is an abstraction step, then $t_{1} \cdot \gamma$ is an abstraction of $t^{\prime} \sigma$ and, hence, we can conclude by the induction hypothesis. If, otherwise, the first is not an abstraction step, then $\left.t \gamma\right|_{p_{1}} \equiv l$ and $t_{1} \gamma \equiv t \gamma[r]_{p_{1}}$ for some rule $l \rightarrow r$ in $\vec{E}$. We can assume there is an equation $l^{\prime} \simeq r^{\prime}$ in $E$ such that $l^{\prime} \sigma \equiv l$ and $r^{\prime} \sigma \equiv r$. Therefore, since $l \succ r$ and $p_{1}$ is a non-variable position of $t^{\prime}$, there is an inference by ordered paramodulation,

$$
\frac{l^{\prime} \simeq r^{\prime} \quad s^{\prime} \simeq t^{\prime}}{\left(s^{\prime} \simeq t^{\prime}\left[r^{\prime}\right]_{p_{1}}\right) \theta}
$$

where $\theta=m g u\left(\left.t^{\prime}\right|_{p_{1}}, l^{\prime}\right)$, whose conclusion has an instance of the form $s^{\prime} \sigma \simeq$ $t^{\prime} \sigma\left[r^{\prime} \sigma\right]_{p_{1}}$, i.e., $s^{\prime} \sigma \simeq t_{1} \gamma$, under the substitution $\sigma$.

Since $E$ is closed under $\mathcal{E}$ and $t_{1} \gamma \not \equiv s^{\prime} \sigma$ by assumption, then the conclusion of the previous inference is in $E$ even if tautologies are deleted. Moreover, we have that $s^{\prime}$ subsumes $s^{\prime} \theta$, and $t^{\prime}[]_{p_{1}}$ subsumes $t^{\prime}[]_{p_{1}} \theta$. Then, for all $i$ in $\{2, \ldots, n\}$, $p_{i}$ is not below any variable position of $t^{\prime}\left[r^{\prime}\right]_{p_{1}} \theta$ (recall that, by definition of $B$-rewriting, no further step can take place below $\left.p_{1}\right)$. Altogether, this lets us conclude by the induction hypothesis.

Lemma 10. Let $E$ be a set of equations closed under $\mathcal{E}$, and $l \simeq r$ and $s \simeq t$ be two equations in $E$ such that $l$ and $s$ are both variables and, for some ground substitution $\sigma$, it holds that $s \sigma \equiv l \sigma \succ r \sigma \not \equiv t \sigma$. Then there is an equation $r^{\prime} \simeq t$ in $E$ with $r^{\prime} \sigma \equiv r \sigma$.

Proof. Recall from Definition 3 that inferences at variable positions are allowed in a special case where $l$ is a variable $x$ and $s$ is a variable $y$. The inference is

$$
\frac{l \simeq r \quad s \simeq t}{r \theta \simeq t},
$$

where $\theta$ is just the variable renaming of $y$ by $x$, and $l$ is maximal in its premise, that is, we have that $l \theta \sigma \equiv l \sigma \succ r \sigma \equiv r \theta \sigma$. Moreover, the conclusion is in $E$ even if tautologies are deleted, since it has an instance of the form $r \theta \sigma \simeq t \sigma$, i.e., $r \sigma \simeq t \sigma$, with $r \sigma \not \equiv t \sigma$. 
Note that, in the previous lemma, we do not require $s \sigma \succ t \sigma$.

Using the previous lemmas we can infer that, for a set of equations $E$ that is closed under $\mathcal{E}$, no righthand side of $R_{E}$ appears as lefthand side neither in $R_{E}$ nor in $R_{E}^{\circ}$, and that there are no two rules neither in $R_{E}$ nor in $R_{E}^{\circ}$ with the same lefthand side.

Definition 10 (Rule introducing a righthand side). Let $R$ be a ground $T R S$, and $t$ a term in Right $(R)$. Then we define the rule introducing $t$ in $R$ as the smallest rule w.r.t. $\succ_{e}$ in $R$ with $t$ as righthand side.

Lemma 11. Let $E$ be a set of equations that is closed under $\mathcal{E}$. Then Left $\left(R_{E}\right) \cap$ $\operatorname{Right}\left(R_{E}\right)=\emptyset$.

Proof. We proceed by contradiction. Let $t$ be the smallest term in $\operatorname{Left}\left(R_{E}\right) \cap$ $\operatorname{Right}\left(R_{E}\right)$ w.r.t. $\succ$. Then there is a rule of the form $t \rightarrow u$ in $R_{E}$, and a rule $s \rightarrow t$ introducing $t$ in $R_{E}$. Note that the two rules are different, since $s \succ t \succ u$ and $\succ$ is well-founded. Now, if $s \simeq t \succ_{e} t \simeq u$, then $t$ is reducible by $R_{E}^{s \simeq t}$ at the topmost position and since, by minimality of $s \simeq t, t \notin \operatorname{Right}\left(R_{E}^{s \simeq t}\right)$, we have that $\lambda$ is a position of $t$ in $\operatorname{Strict} C o n t e x t\left(t, \operatorname{Right}\left(R_{E}^{s \simeq t}\right)\right)$. But this contradicts Lemma 4. Therefore we necessarily have that $t \simeq u \succ_{e} s \simeq t$, and hence $|t| \geq|s|$.

Let $s^{\prime} \simeq t^{\prime}$ and $t^{\prime \prime} \simeq u^{\prime}$ be two equations in $E$ such that, for some ground substitution $\sigma$, it holds that $s^{\prime} \sigma \equiv s, t^{\prime} \sigma \equiv t^{\prime \prime} \sigma \equiv t$ and $u^{\prime} \sigma \equiv u$. Observe that, if $t^{\prime}$ is a variable $x$, then $x$ must occur in $s^{\prime}$ since, otherwise, the theory is trivial. But, since $s \not \equiv t$, having $t^{\prime} \equiv x$ and $x$ occurring in $s^{\prime}$ contradicts $|t| \geq|s|$.

Therefore, $t^{\prime}$ cannot be a variable. Hence, since $t \rightarrow u \in R_{E}$ and $R_{E} \subseteq R_{E}^{\circ}$, we have that

$$
t \curvearrowright{\stackrel{\lambda}{R_{E}^{\circ}}}^{\lambda} z \cdot\{z \mapsto u\},
$$

where $\lambda$ is not below any variable position of $t^{\prime}$. And $u \not \equiv s$, since we have that $s \succ t \succ u$ and $\succ$ is well-founded. Then, by Lemma 9, there is an equation $s^{\prime \prime} \simeq u^{\prime \prime}$ in $E$, where $s^{\prime \prime}$ is subsumed by $s^{\prime}$, that has an instance of the form $s \simeq u$. And, since $t \succ u$, we have that $s \simeq t \succ_{e} s \simeq u$.

Now recall that $s \rightarrow t \in R_{E}$ and hence, by minimality of $t$ w.r.t. $\succ$, we have that $s \notin \operatorname{Right}\left(R_{E}\right)$. This fact gives us that $s \simeq u$ cannot generate the rule $s \rightarrow u$ in $R_{E}$ : otherwise, as $s \notin \operatorname{Right}\left(R_{E}\right)$, this would be a contradiction with Lemma 7 for $s \rightarrow t$. Let us now analyze the possible reasons why $s \simeq u$ does not generate a rule in $R_{E}$. Since $s \succ u$, we have the following two possibilities left:

1. $s$ is reducible by $R_{E}^{s \simeq u}$ at some position in $\operatorname{Strict} \operatorname{Context}\left(s, \operatorname{Right}\left(R_{E}^{s \simeq u}\right)\right)$ and hence, by Lemma 3 , at $\operatorname{Strict} \operatorname{Context}\left(s, \operatorname{Right}\left(R_{E}\right)\right)$. Moreover, since $s \simeq t \succ_{e} s \simeq u$, we have that $s \rightarrow t \notin R_{E}^{s \simeq u}$. Therefore, $s$ is reducible by $R_{E} \backslash$ $\{s \rightarrow t\}$ at some position in StrictContext $\left(s, \operatorname{Right}\left(R_{E}^{s \simeq t}\right)\right)$, which contradicts Lemma 7 again (note that $R_{E}^{s \simeq t} \subseteq R_{E}$ implies $\operatorname{Strict} \operatorname{Context}\left(s, \operatorname{Right}\left(R_{E}\right)\right) \subseteq$ StrictContext $\left.\left(s, \operatorname{Right}\left(R_{E}^{s \simeq t}\right)\right)\right)$.

2. $u$ is reducible by $R_{E}^{s \simeq u}$ at some position in $\operatorname{Strict} \operatorname{Context}\left(u, \operatorname{Right}\left(R_{E}^{s \simeq u}\right)\right)$. Then, since $t \rightarrow u \in R_{E}$ and hence $u \in \operatorname{Right}\left(R_{E}\right)$, this would be a contradiction with Lemma 4. 
Lemma 12. Let $E$ be a set of equations that is closed under $\mathcal{E}$. Then Left $\left(R_{E}^{\circ}\right) \cap$ $\operatorname{Right}\left(R_{E}\right)=\emptyset$.

Proof. We proceed by contradiction. Assume that $t$ is a term in $\operatorname{Right}\left(R_{E}\right)$ and there is some rule of the form $t \rightarrow u$ in $R_{E}^{\circ}$. Since $t$ is a term in $\operatorname{Right}\left(R_{E}\right)$, we have that $t \notin \operatorname{Left}\left(R_{E}\right)$ by Lemma 11 . Then $t \rightarrow u \in R_{E}^{\circ} \backslash R_{E}$. But then, by Lemma 5 , we have that $t$ is reducible by $R_{E}$ at $\operatorname{Strict} \operatorname{Context}\left(t, \operatorname{Right}\left(R_{E}^{t \sim u}\right)\right)$, contradicting $t \in \operatorname{Right}\left(R_{E}\right)$ (note that, if $t \in \operatorname{Right}\left(R_{E}\right)$, then $\operatorname{Strict} \operatorname{Context}\left(t, \operatorname{Right}\left(R_{E}^{t \simeq u}\right)\right.$ ) is empty).

Lemma 13. Let $E$ be a set of equations that is closed under $\mathcal{E}$. Then there are no two rules in $R_{E}$ with the same lefthand side.

Proof. If $E$ is a set of equations that is closed under $\mathcal{E}$ then, by Lemma 11, we have that $\operatorname{Left}\left(R_{E}\right) \cap \operatorname{Right}\left(R_{E}\right)=\emptyset$. Then $\lambda$ is in $\operatorname{Strict} \operatorname{Context}\left(s, \operatorname{Right}\left(R_{E}\right)\right)$ for every rule $s \rightarrow t$ in $R_{E}$ and, hence, by Lemma 7 , there cannot be two different rules in $R_{E}$ with the same lefthand side.

Lemma 14. Let $E$ be a set of equations that is closed under $\mathcal{E}$. Then there are no two rules in $R_{E}^{\circ}$ with the same lefthand side.

Proof. Assume the contrary, i.e., that there are two rules of the form $l \rightarrow r$ and $l \rightarrow t$ in $R_{E}^{\circ}$. Then, there are two equations $l^{\prime} \simeq r^{\prime}$ and $l^{\prime \prime} \simeq t^{\prime}$ in $E$ such that, for some ground substitution $\sigma$, we have $l^{\prime} \sigma \equiv l^{\prime \prime} \sigma \equiv l, r^{\prime} \sigma \equiv r$ and $t^{\prime} \sigma \equiv t$. We distinguish between the following situations:

1. $l^{\prime}$ is not a variable. Then

$$
l \curvearrowright_{\{l \rightarrow t\}}^{\lambda} x \cdot\{x \mapsto t\},
$$

where $\lambda$ is not a below any variable position of $l^{\prime}$ and $t \not \equiv r^{\prime} \sigma$. Then, by Lemma 9 applied on $r^{\prime} \simeq l^{\prime}$, there is an equation in $\operatorname{gnd}(E)$ of the form $r^{\prime} \sigma \simeq t$, i.e., $r \simeq t$. Since both $r$ and $t$ belong to $\operatorname{Right}\left(R_{E}\right)$ then, regardless of the orientation of $r \simeq t$ w.r.t. $\succ$, we will have a rule in $R_{E}^{\circ}$ whose lefthand side is in $\operatorname{Right}\left(R_{E}\right)$, contradicting Lemma 12.

2. $l^{\prime \prime}$ is not a variable. Then

$$
l \curvearrowright_{\{l \rightarrow r\}}^{\lambda} x \cdot\{x \mapsto r\},
$$

where $\lambda$ is not a below any variable position of $l^{\prime \prime}$ and $r \not \equiv t^{\prime} \sigma$. Then, by Lemma 9 applied on $t^{\prime} \simeq l^{\prime \prime}$, there is an equation in $\operatorname{gnd}(E)$ of the form $t^{\prime} \sigma \simeq r$, i.e., $t \simeq r$, and we conclude as in the previous case.

3. $l^{\prime}$ and $l^{\prime \prime}$ are variables. Then, as $l^{\prime \prime} \sigma \equiv l^{\prime} \sigma \succ r^{\prime} \sigma \not \equiv t^{\prime} \sigma$, by Lemma 10 we have that $r^{\prime} \sigma \simeq t^{\prime} \sigma$, i.e., $r \simeq t$, is in $\operatorname{gnd}(E)$, and we conclude as in the previous cases. 


\subsection{The Return Property}

In this subsection we prove the so-called Return Property of $R_{E}$, which roughly states that, if we reduce, with $R_{E}$, a righthand side $t$ of $R_{E}$ into a term $u$, then there is a rewrite sequence with $R_{E}$ from $u$ to $t$ again. This strong property is crucial to prove that our generated $R_{E}$, in spite of being potentially overlapping and non-terminating, is confluent: after the Return Property is proved, confluence follows from the fact that the lefthand sides only overlap below subterms corresponding to righthand sides. See Figure 3 for an example.

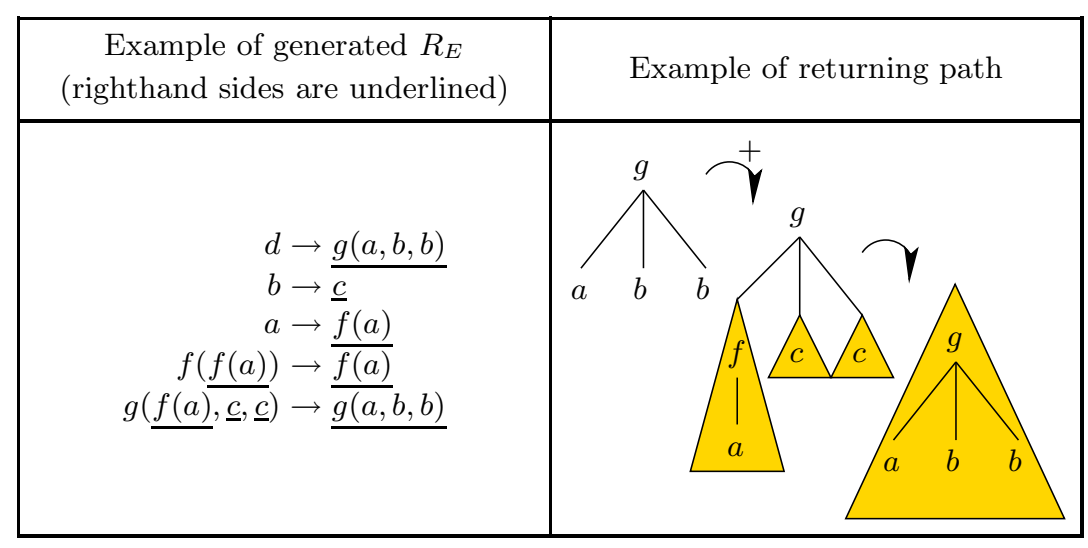

Figure 3. Illustration of the Return Property.

The intuition behind this property comes from the fact that the reducible righthand sides of $R_{E}$ are minimal w.r.t. $\succ$ in their congruence class. Hence, for the ground case, if a righthand side $t$ is reducible at some position $p$ by some rule $l \rightarrow r$, then there is an inference by ordered paramodulation between a pair of equations $l \simeq r$ and $s \simeq t$ leading to $s \simeq t[r]_{p}$ (since $l \succ r$ ) and, again, another inference between $s \simeq t$ and $s \simeq t[r]_{p}$ leading to $t \simeq t[r]_{p}$ (since $s \succ t$ ), which lets us to get back to $t$ with $t[r]_{p} \rightarrow t$ (since $t[r]_{p} \succ t$ ).

Following the example, if we have $a \simeq f(a)$ with $f(a)$ minimal and $f(a)$ reducible by $a \rightarrow f(a)$, we can obtain $a \simeq f(f(a))$ and then $f(f(a)) \simeq f(a)$ by an inference at the topmost position, with $f(f(a)) \succ f(a)$. Thus, after reducing $f(a)$ into $f(f(a))$ with $a \rightarrow f(a)$, we can return to $f(a)$ using $f(f(a)) \rightarrow f(a)$.

Thanks to this Return Property, $t$ can be chosen as a representative (not a normal form, since it can be reducible) of its congruence class. Moreover, for all terms in its congruence class, their representative can be obtained by an innermost rewrite sequence consisting in replacing every reducible subterm by its representative.

However, for the non-ground case, the proof becomes rather technical due to the side effects of reductions that correspond to ground inferences that take place below non-linear variables, which cannot be lifted to non-ground inferences. 
Now we introduce some new lemmas, concepts and notation, which are used in the proof of the Return Property.

The following lemma states that, in a sequence by $B$-rewriting with a TRS $R$, if some step has taken place at the border, w.r.t. $\operatorname{Right}(R)$, of the initial term, then it is not possible to get back to the initial term until a normal form has been reached.

Lemma 15. Let $R$ be a ground TRS and $t \cdot \gamma$ an abstracted term. If $t \cdot \gamma \curvearrowright_{R}^{p_{1}}$ $t_{1} \cdot \gamma \cdots \curvearrowright_{R}^{p_{n}} t_{n} \cdot \gamma$, where $n \geq 1, p_{1} \in \operatorname{Border}(t \gamma, \operatorname{Right}(R))$ and $t_{n} \cdot \gamma$ is not abstracted at the topmost position, then $t_{i} \gamma \not \equiv t \gamma$ for all $i$ in $\{1, \ldots, n\}$.

Proof. If $t \cdot \gamma \curvearrowright_{R}^{p_{1}} t_{1} \cdot \gamma \cdots \curvearrowright_{R}^{p_{i}} t_{i} \cdot \gamma$, where $i \geq 1$, then, by definition of $B$ rewriting, $p_{1}$ is below some position $p$ such that $\left.t_{i} \gamma\right|_{p} \in \operatorname{Right}(R)$. Moreover, if $t_{i} \cdot \gamma$ is not abstracted at the topmost position (as is the case if $t_{i} \cdot \gamma \curvearrowright_{R}^{*} t_{n} \cdot \gamma$ and $t_{n} \cdot \gamma$ is not abstracted at the topmost position), then there is such a position $p$ which is not topmost, i.e., $p \neq \lambda$. Therefore, $p_{1} \notin \operatorname{Border}\left(t_{i} \gamma, \operatorname{Right}(R)\right)$. Finally since, by assumption, $p_{1} \in \operatorname{Border}(t \gamma, \operatorname{Right}(R))$, we necessarily have that $t_{i} \gamma \not \equiv t \gamma$.

We define two restricted versions of the $B$-rewrite relation $\curvearrowright_{R}$, denoted by $\curvearrowright_{\underline{R}}$ and $\curvearrowright_{\bar{R}}$, which respectively consist of rewriting only above blocked positions and only above non-blocked positions. Recall from Definition 2 that steps below ${ }^{5}$ blocked positions are forbidden for $\curvearrowright_{R}$. Then, for example, in the abstracted term $f(a, x) \cdot\{x \mapsto g(a)\}$, we can rewrite with $\curvearrowright_{\underline{R}}$ only at position $\lambda$ and with $\curvearrowright_{\bar{R}}$ only at position 1 .

Definition 11 (Restricted $B$-rewriting). Let $s \cdot \gamma$ and $t \cdot \delta$ be two abstracted terms, $R$ be a ground TRS and $p$ be a position. Then

$$
s \cdot \gamma \curvearrowright_{\underline{R}}^{p} t \cdot \delta \quad \text { iff } s \cdot \gamma \curvearrowright_{R}^{p} t \cdot \delta \text { by a non-abstraction step and } \operatorname{Var}\left(\left.s\right|_{p}\right) \neq \emptyset,
$$

and

$$
s \cdot \gamma \curvearrowright \frac{p}{R} t \cdot \delta \quad \text { iff } \quad s \cdot \gamma \curvearrowright_{R}^{p} t \cdot \delta \text { by a non-abstraction step and } \operatorname{Var}\left(\left.s\right|_{p}\right)=\emptyset .
$$

Note that one relation is the complement of the other if we restrict to nonabstraction steps.

Definition 12 (Innermost $B$-rewriting). Given a ground TRS $R$ and two abstracted terms $s \cdot \gamma$ and $t \cdot \delta$, we write

$$
s \cdot \gamma \curvearrowright_{R}^{i p} t \cdot \delta \quad \text { iff }
$$

$s \cdot \gamma \curvearrowright_{R}^{p} t \cdot \delta$ for some deepest reducible position $p$ of $s \cdot \gamma$ by $\curvearrowright_{R}$. We may often write $s \cdot \gamma \curvearrowright_{R}^{i} t \cdot \delta$ when the position $p$ is not relevant or clear from the context.

\footnotetext{
${ }^{5}$ We speak of positions being above and below in a non strict way (see Section 2).
} 
The previous definition is extended to the case of restricted $B$-rewriting in the obvious way. If we do not have different rules with the same lefthand side we have the following innermost confluence result:

Lemma 16. Let $R$ and $S$ be two sets of rules, such that there are no two rules neither in $R$ nor in $S$ with the same lefthand side. Then, both

$$
\curvearrowright_{R}^{i} \text { and }\left(\curvearrowright_{S} \cup \curvearrowright_{\underline{R}}\right)^{i} \text { are confluent. }
$$

Proof. Since there are no two rules neither in $R$ nor in $S$ with the same lefthand side, and, by definition, all steps with $\curvearrowright_{\bar{S}}$ and $\curvearrowright_{\underline{R}}$ are disjoint, we have that all rewriting steps with $\curvearrowright_{R}^{i}$ and $\left(\curvearrowright_{\bar{S}} \cup \curvearrowright_{\underline{R}}\right)^{i}$ are non-overlapping. Therefore, they are trivially locally confluent and, since both relations are terminating, confluence holds.

The following definition states a particular kind of return property, that we assume to hold on some (smaller) terms in our proofs by induction.

Definition 13 (Innermost returning term). Let $E$ be a set of equations that is closed under $\mathcal{E}$. We say that a ground term $t$ is innermost returning iff, for every non-overlapping subset $S$ of $R_{E}^{\circ}$, the normal form $u \cdot \delta$ of $t$ w.r.t. $\left(\curvearrowright_{\bar{S}} \cup \curvearrowright_{\underline{R_{E}^{\circ}}}\right)^{i}$ fulfills $u \delta \equiv t$.

The following ordering is defined on righthand sides of $R_{E}$ that are reducible in the border, and it is crucial for proving the forthcoming lemmas.

Definition $14\left(\succ_{B}\right)$. Let $B$ be the set of terms $t$ such that $t \in \operatorname{Right}\left(R_{E}\right)$ and $t$ is reducible by $R_{E}^{\circ}$ at some position in Border $\left(t, \operatorname{Right}\left(R_{E}\right)\right)$. Then, for every pair of terms $s$ and $t$ in $B$, we have that $s \succ_{B} t$ if and only if either $s \triangleright t$ or the rule $l \rightarrow s$ introducing $s$ in $R_{E}$ satisfies $l \triangleright t$.

Lemma 17. The relation $\succ_{B}$ is well-founded on $B$.

Proof. Consider the ordering $\succ_{r}$ on $\operatorname{Right}\left(R_{E}\right)$ defined as $s \succ_{r} t$ iff $l \rightarrow s$ is the rule introducing $s$ in $R_{E}, l^{\prime} \rightarrow t$ is the rule introducing $t$ in $R_{E}$ and $l \simeq s \succ_{e} l^{\prime} \simeq t$. Note that, since $\succ_{e}$ is well-founded, we have $\succ_{r}$ well-founded and hence its multiset extension $\succ_{r}$ on finite multisets of terms in $\operatorname{Right}\left(R_{E}\right)$ is well-founded too. Finally, let $M(t)$ be defined as the multiset containing all terms $t^{\prime} \in B$, such that $t \unrhd t^{\prime}$. Now, we show that if $s \succ_{B} t$ then $M(s) \succ_{r} M(t)$, which implies that $\succ_{B}$ is well-founded.

If $s \succ_{B} t$ because $s \triangleright t$, then, as $M(s) \supset M(t)$, we have that $M(s) \succ_{r} M(t)$. Otherwise, we have that the rule $l \rightarrow s$ introducing $s$ in $R_{E}$ satisfies $l \triangleright t$. In this case, we prove that for all $v \in M(t)$ we have $s \succ_{r} v$ which, since $s \in M(s)$, implies that $M(s) \succ_{r} M(t)$.

If $v \in M(t)$ then $l \triangleright v$ and $v \in B$ which, by Lemma 6 , implies that there is some rule $l^{\prime} \rightarrow r^{\prime}$ in $R_{E}$ reducing $v$ at $\operatorname{Border}\left(v, \operatorname{Right}\left(R_{E}\right)\right)$. Since $l \triangleright l^{\prime}$ we have that $l \simeq s \succ_{e} l^{\prime} \simeq r^{\prime}$. On the other hand, if $l^{\prime \prime} \rightarrow v$ is the rule introducing $v$, then $l^{\prime} \simeq r^{\prime} \succ_{e} l^{\prime \prime} \simeq v$ since, otherwise, $v$ would be reducible by 
$R_{E}^{l^{\prime \prime} \simeq v}$ at $\operatorname{Border}\left(v, \operatorname{Right}\left(R_{E}\right)\right) \subseteq \operatorname{Strict} \operatorname{Context}\left(v, \operatorname{Right}\left(R_{E}^{l^{\prime \prime} \simeq v}\right)\right)$, contradicting Lemma 4. Finally, since $l \simeq s \succ_{e} l^{\prime} \simeq r^{\prime} \succ_{e} l^{\prime \prime} \simeq v$, we have that $l \simeq s \succ_{e} l^{\prime \prime} \simeq v$.

Lemma 22 is the central lemma of our result. Here follow some auxiliary lemmas used in its proof.

Lemma 18. Let $E$ be a set of equations that is closed under $\mathcal{E}$ and $S$ be a nonoverlapping subset of $R_{E}^{\circ}$. Let $t \cdot \gamma$ and $t^{\prime} \cdot \gamma^{\prime}$ be two abstracted terms such that $t \gamma \equiv t^{\prime} \gamma^{\prime}$, all non-variable positions of $t^{\prime}$ are non-variable positions of $t$, and every position of $t \cdot \gamma$ reducible by $\left(\curvearrowright_{\bar{S}} \cup \curvearrowright_{R_{E}^{\circ}}\right)^{i}$ is a position of $t^{\prime} \cdot \gamma^{\prime}$ reducible by $\left(\curvearrowright_{\bar{S}} \cup \curvearrowright_{\underline{R_{E}^{\circ}}}\right)^{i}$. Then

$$
t \cdot \gamma\left(\curvearrowright_{\bar{S}} \cup \curvearrowright_{\underline{R_{E}^{\circ}}}\right)^{i *} u \cdot \delta
$$

implies

$$
t^{\prime} \cdot \gamma^{\prime}\left(\curvearrowright_{\bar{S}} \cup \curvearrowright_{\underline{R_{E}^{\circ}}}\right)^{i *} v \cdot \rho,
$$

with the same steps, where $u \delta \equiv v \rho$ and all non-variable positions of $v$ are nonvariable positions of $u$.

Proof. We proceed by induction on $\left(\curvearrowright_{\bar{S}} \cup \curvearrowright_{R_{E}^{\circ}}\right)^{i}$. If there is no rewrite step on $t \cdot \gamma$, then $t \cdot \gamma \equiv u \cdot \delta$ and, since $t \gamma \equiv t^{\prime} \gamma^{\prime}$ and $\overline{\text { all }}$ non-variable positions of $t^{\prime}$ are non-variable positions of $t$, we can take $v \cdot \rho \equiv t^{\prime} \cdot \gamma^{\prime}$.

Otherwise, let $p$ be the first position of $t \cdot \gamma$ that we are reducing. Then, by assumption, $p$ is a reducible position of $t^{\prime} \cdot \gamma^{\prime}$. Since $t \gamma \equiv t^{\prime} \gamma^{\prime}$, we have that $\left.\left.t\right|_{p} \gamma \equiv t^{\prime}\right|_{p} \gamma^{\prime}$. Now, by Lemma 14, there is a single rule in $R_{E}^{\circ}$ with $\left.t\right|_{p} \gamma$ as lefthand side. Then, using the rule $\left.t\right|_{p} \gamma \rightarrow r$, we have

$$
t \cdot \gamma\left(\curvearrowright_{S} \cup \curvearrowright_{\underline{R_{E}^{\circ}}}\right)^{i} t[y]_{p} \cdot(\gamma \cup\{y \mapsto r\})\left(\curvearrowright_{\bar{S}} \cup \curvearrowright_{\underline{R_{E}^{\circ}}}\right)^{i *} u \cdot \delta
$$

and also

$$
t^{\prime} \cdot \gamma^{\prime}\left(\curvearrowright_{S} \cup \curvearrowright_{\underline{R_{E}^{\circ}}}\right)^{i} t^{\prime}[y]_{p} \cdot\left(\gamma^{\prime} \cup\{y \mapsto r\}\right) .
$$

Now, let $\gamma_{1}=\gamma \cup\{y \mapsto r\}$ and $\gamma_{1}^{\prime}=\gamma^{\prime} \cup\{y \mapsto r\}$. We have:

$-t[y]_{p} \gamma_{1} \equiv t^{\prime}[y]_{p} \gamma_{1}^{\prime}$

- All non-variable positions of $t^{\prime}[y]_{p}$ are non-variable positions of $t[y]_{p}$, since $p$ is a variable position of both terms and all other non-variable positions of $t^{\prime}[y]_{p}$ are non-variable positions of $t^{\prime}$ not below $p$ and thus, by assumption, non-variable positions of $t$ not below $p$, and hence non-variable positions of $t[y]_{p}$.

- Every position of $t[y]_{p} \cdot \gamma_{1}$ reducible by $\left(\curvearrowright_{S} \cup \curvearrowright_{R_{E}^{\circ}}\right)^{i}$ is a reducible position of $t^{\prime}[y]_{p} \cdot \gamma_{1}^{\prime}$, since all new reducible positions in $\bar{t}[y]_{p} \cdot \gamma_{1}$ are reducible by $\curvearrowright_{\underline{R_{E}^{\circ}}}$ above $p$, and hence they are also reducible by $\curvearrowright_{\underline{R_{E}^{\circ}}}$ above $p$ in $t^{\prime}[y]_{p} \cdot \gamma_{1}^{\prime}$. 
Therefore, by the induction hypothesis, we have

$$
t^{\prime}[y]_{p} \cdot \gamma_{1}^{\prime}\left(\curvearrowright_{\bar{S}} \cup \curvearrowright_{\underline{R_{E}^{\circ}}}\right)^{i *} v \cdot \rho,
$$

where $u \delta \equiv v \rho$ and all non-variable positions of $v$ are non-variable positions of $u$.

Lemma 19. Let $E$ be a set of equations that is closed under $\mathcal{E}, t$ be a term in $\operatorname{Right}\left(R_{E}\right)$, and $s \rightarrow t$ be the rule in $R_{E}$ introducing ${ }^{6} t$. If some rule $l \rightarrow r$ in $R_{E}^{\circ}$ reduces $t$ at some position in Border $\left(t, \operatorname{Right}\left(R_{E}\right)\right)$, then either $l$ does not occur in $s$, or $l \rightarrow r$ and $s \rightarrow t$ are the same rule.

Proof. Assume that some rule $l \rightarrow r$ in $R_{E}^{\circ}$ reduces $t$ at some position $p$ in $\operatorname{Border}\left(t, \operatorname{Right}\left(R_{E}\right)\right)$, i.e., at $\operatorname{Strict} \operatorname{Context}\left(t, \operatorname{Right}\left(R_{E}\right) \backslash\{t\}\right)$.

First of all note that, since $s \rightarrow t$ is the rule in $R_{E}$ introducing $t$, we have that $t \notin \operatorname{Right}\left(R_{E}^{s \simeq t}\right)$ and, hence, $\operatorname{Right}\left(R_{E}^{s \simeq t}\right) \subseteq \operatorname{Right}\left(R_{E}\right) \backslash\{t\}$. Therefore, $\operatorname{StrictContext}\left(t, \operatorname{Right}\left(R_{E}\right) \backslash\{t\}\right) \subseteq \operatorname{Strict} \operatorname{Context}\left(t, \operatorname{Right}\left(R_{E}^{s \simeq t}\right)\right)$.

Now, either $l \rightarrow r \in R_{E}$ or, by Lemma 5 , we have that $l$ is reducible by $R_{E}^{l \sim r}$ at some position in $\operatorname{Strict} \operatorname{Context}\left(l, \operatorname{Right}\left(R_{E}^{l \simeq r}\right)\right)$ and, hence, by Lemma 3, in $\operatorname{Strict} \operatorname{Context}\left(l, \operatorname{Right}\left(R_{E}\right)\right)$. Therefore, in any case, if $s \simeq t \succ_{e} l \simeq r$, then some rule in $R_{E}^{s \simeq t}$ would reduce $t$ below $p$, a position in StrictContext $\left(t, \operatorname{Right}\left(R_{E}^{s \simeq t}\right)\right)$, contradicting Lemma 4. Therefore, we necessarily have that $l \simeq r \succeq_{e} s \simeq t$ and, hence, $|l|>=|s|$. Finally since, by Lemma 14 , there are no two different rules in $R_{E}^{\circ}$ with the same lefthand side, we have that either $l$ does not occur in $s$, or $l \rightarrow r$ and $s \rightarrow t$ are the same rule.

Lemma 20. Let $E$ be a set of equations that is closed under $\mathcal{E}, t$ be a term in Right $\left(R_{E}\right)$, and $s \rightarrow t$ be the rule in $R_{E}$ introducing $t$. If $s$ and $t$ differ only below some position $p$ in Border $\left(t, \operatorname{Right}\left(R_{E}\right)\right)$ then, for every reducible position $q$ of $s$ by $R_{E}^{\circ}$ such that $q$ is not overlapped with $p$, there is a position $q^{\prime}$ above $q$ such that $\left.s\right|_{q^{\prime}} \in \operatorname{Right}\left(R_{E}\right)$ and $q^{\prime}$ is not overlapped with $p$.

Proof. Let $u \rightarrow v$ be the rule in $R_{E}^{\circ}$ reducing $s$ at position $q$. Since $q$ is not overlapped with $p$, then $q \neq \lambda$, i.e., $u$ occurs at a non-topmost position of $s$. Moreover, as $s$ and $t$ differ only at positions below $p$, we have that $u \rightarrow v$ also reduces $t$ at position $q$. Then, by Lemma 19, we have that $q \notin \operatorname{Border}\left(t, \operatorname{Right}\left(R_{E}\right)\right)$, i.e., there is a position $q^{\prime} \neq \lambda$ above $q$ such that $\left.t\right|_{q^{\prime}} \in \operatorname{Right}\left(R_{E}\right)$. Moreover, as $p \in \operatorname{Border}\left(t, \operatorname{Right}\left(R_{E}\right)\right), q^{\prime}$ cannot be above $p$. And, since $s$ and $t$ differ only below $p$, we have that $\left.\left.s\right|_{q^{\prime}} \equiv t\right|_{q^{\prime}}$ and, hence, $\left.s\right|_{q^{\prime}} \in \operatorname{Right}\left(R_{E}\right)$.

Lemma 21. Let $E$ be a set of equations that is closed under $\mathcal{E}, t$ be a term in $\operatorname{Right}\left(R_{E}\right)$, and $s \rightarrow t$ be the rule in $R_{E}$ introducing $t$. If there are two equations $s^{\prime} \simeq t^{\prime}$ and $s^{\prime \prime} \simeq t^{\prime \prime}$ in $E$ such that neither $t^{\prime}$ nor $t^{\prime \prime}$ are variables and, for some substitution $\sigma$ we have that $s^{\prime} \sigma \equiv s, t^{\prime} \sigma \equiv t, s^{\prime \prime} \sigma \equiv t$ and $t^{\prime \prime} \sigma \equiv u$, where $t \not \equiv u$ and the only reducible positions of $u$ by $R_{E}$ belonging to $\operatorname{Border}\left(u, \operatorname{Right}\left(R_{E}\right)\right)$ are either $\lambda$, or positions $p$ of $t$ such that $\left.\left.u\right|_{p} \equiv t\right|_{p}$ and $p \in \operatorname{Border}\left(t, \operatorname{Right}\left(R_{E}\right)\right)$, then $u \succ t$.

\footnotetext{
${ }^{6}$ See Definition 10.
} 
Proof. By assumption, we have that $t \not \equiv u$. We will show that having $t \succ u$ leads to a contradiction and, hence, $u \succ t$.

If $t \succ u$ then, since $s \succ t \succ u$ and $\succ$ is well-founded, we would have that $s \not \equiv u$. Therefore, since $t^{\prime}$ is not a variable and $t \curvearrowright_{\vec{E}} x \cdot\{x \mapsto u\}$ for some variable $x$ then, by Lemma 9 applied on $s^{\prime} \simeq t^{\prime}$, there would be an equation in $E$ with an instance of the form $s \simeq u$.

By Lemma 13, we know that $s \rightarrow u \notin R_{E}$. Let us analyze the conditions required for an equation to generate a rule in $R_{E}$ (see Definition 7 ). Condition 1 is clearly fulfilled, since $s \succ u$. Condition 2 must also be fulfilled since, if $s \simeq u$ fails Condition 2 then, by Lemma 3, also fails $s \simeq t$, as $s \simeq t \succ_{e} s \simeq u$. Hence, $s \simeq u$ must fail Condition 3, i.e., $u$ must be reducible $R_{E}^{s \simeq u}$ at some position $p$ in StrictContext $\left(u, \operatorname{Right}\left(R_{E}^{s \simeq u}\right)\right)$. Then, by Lemma 3, $p$ is also in $\operatorname{Strict} \operatorname{Context}\left(u, \operatorname{Right}\left(R_{E}\right)\right)$, and hence in $\operatorname{Border}\left(u, \operatorname{Right}\left(R_{E}\right)\right)$. And, as seen before, either $p=\lambda$, or $p$ is in $\operatorname{Border}\left(t, \operatorname{Right}\left(R_{E}\right)\right)$ and $\left.\left.u\right|_{p} \equiv t\right|_{p}$.

We can see that in any case we get a contradiction. If $p=\lambda$, then there would be a rule of the form $u \rightarrow v$ in $R_{E}$. Moreover, $t \not \equiv v$, since $t \succ u \succ v$. And, by assumption, $s^{\prime \prime} \simeq t^{\prime \prime}$ is an equation in $E$, where $t^{\prime \prime}$ is not a variable, that has an instance of the form $t \simeq u$. Therefore, by Lemma 9 applied on $s^{\prime \prime} \simeq t^{\prime \prime}$, there would be an equation in $E$ having an instance of the form $t \simeq v$ and, since $t \succ v$, then $t \rightarrow v \in R_{E}^{\circ}$. But, since $t$ belongs to $\operatorname{Right}\left(R_{E}\right)$, this contradicts Lemma 12 .

If $p$ is in $\operatorname{Border}\left(t, \operatorname{Right}\left(R_{E}\right)\right)$ then, since $s \simeq t \succ_{e} s \simeq u$ and $s \rightarrow t$ is the rule introducing $t$ in $R_{E}, p$ is also in $\operatorname{Strict} \operatorname{Context}\left(t, \operatorname{Right}\left(R_{E}^{s \simeq t}\right)\right)$. Since, moreover, $\left.\left.u\right|_{p} \equiv t\right|_{p}$, we have that $t$ is reducible by $R_{E}^{s \simeq u}$, and hence by $R_{E}^{s \simeq t}$, at position $p$, leading to a contradiction with Lemma 4.

Now we prove the main lemma in which the Return Property is based.

Lemma 22. Let $E$ be a set of equations that is closed under $\mathcal{E}$, $t$ be a term in $\operatorname{Right}\left(R_{E}\right)$, and $S$ be a non-empty and non-overlapping subset of $R_{E}^{\circ}$ such that every rule in $S$ reduces $t$ at some position in Border $\left(t, \operatorname{Right}\left(R_{E}\right)\right)$. If all terms smaller than $t$ w.r.t. $\succ_{B}$ are innermost returning, then we have that

$$
t\left(\curvearrowright_{S} \cup \curvearrowright_{\underline{R_{E}^{\circ}}}\right)^{i !} x \cdot\{x \mapsto t\}
$$

for some variable $x$.

Proof. Let us define

$$
\curvearrowright=\left(\curvearrowright_{S} \cup \curvearrowright_{\underline{R_{E}^{\circ}}}\right)^{i}
$$

In order to proof the lemma we build a derivation

$$
D=t_{0} \cdot \gamma \curvearrowright^{p_{1}} t_{1} \cdot \gamma \cdots \curvearrowright^{p_{n}} t_{n} \cdot \gamma
$$

with $\curvearrowright$, where $p_{1}, \ldots, p_{n}$ denote the positions where the rewrite steps take place, $t_{0} \equiv t$ (and, since $t$ is ground, $t_{0} \cdot \gamma \equiv t$ ) and $t_{n} \cdot \gamma \equiv x \cdot\{x \mapsto t\}$.

Now let $s \rightarrow t$ be the rule introducing $t$ in $R_{E}$, and $s^{\prime} \simeq t^{\prime}$ be an equation in $E$ such that $s^{\prime} \sigma \equiv s$ and $t^{\prime} \sigma \equiv t$ for some ground substitution $\sigma$. We can 
show that $t^{\prime}$ cannot be a variable: if $t^{\prime}$ is a variable $x$ then, either we have a trivial theory, which we assume is not the case, or $x$ must necessarily occur in $s^{\prime}$. In particular, $x$ must occur at some non-topmost position of $s^{\prime}$, since we have that $s^{\prime} \sigma \succ t^{\prime} \sigma$. But then, for every rule $l \rightarrow r$ in $S$, we would have that $s \triangleright l$, contradicting Lemma 19.

We consider $\sigma^{\prime}$ to be a normalization of $\sigma$ w.r.t. $\curvearrowright$, but without keeping the blockings, i.e., a ground substitution $\sigma^{\prime}$ such that $x \sigma ! \curvearrowright$ is an abstraction of $x \sigma^{\prime}$ for every variable $x$. Then there is some $t_{k}$, such that we can start $D$ with

$$
t_{0} \cdot \gamma \curvearrowright^{p_{1}} t_{1} \cdot \gamma \cdots \curvearrowright^{p_{k}} t_{k} \cdot \gamma
$$

and $t_{k} \gamma \equiv t^{\prime} \sigma^{\prime}$ (note that, in particular, if $x \sigma$ is irreducible by $S$ for all variables $x$ in $\operatorname{Var}\left(t^{\prime}\right)$, then $t^{\prime} \sigma^{\prime} \equiv t^{\prime} \sigma$ and $\left.k=0\right)$.

Then, from $t_{k} \cdot \gamma$ on, all remaining steps to be made in $D$ must take place at some position which is not below any variable position of $t^{\prime}$. Observe also that $t_{k} \cdot \gamma$ is not abstracted at the topmost position, since this could only be possible if $t^{\prime}$ was a variable, which, as we have seen, is not the case. Moreover, either $s^{\prime} \sigma^{\prime} \equiv s^{\prime} \sigma \equiv s$, or $s^{\prime} \sigma^{\prime} \equiv t$ since, by Lemma 19 , for every rule $l \rightarrow r$ in $S$, either $l$ does not occur in $s$, or $l \rightarrow r$ and $s \rightarrow t$ are the same rule. We distinguish between the following situations:

1. Some step with $\curvearrowright_{\bar{S}}$ has already taken place in $D$ at some position in $\operatorname{Border}\left(t, \operatorname{Right}\left(R_{E}\right)^{S}\right)$ when we reach $t_{k} \cdot \gamma$. We can assume, w.l.o.g., that the step with $\curvearrowright_{\bar{S}}$ at the border has been the first one, i.e. at position $p_{1}$. In this case, let $t_{m} \cdot \gamma$ be an abstracted term obtained in a derivation with $\curvearrowright$ starting with $t_{k} \cdot \gamma$, and reducing at all positions but $\lambda$. Then, we have

$$
t_{k} \cdot \gamma \curvearrowright^{p_{k+1}} t_{k+1} \cdot \gamma \cdots \curvearrowright^{p_{m}} t_{m} \cdot \gamma
$$

where $t_{m} \cdot \gamma$ is not abstracted at the topmost position and if it is not in normal form then it is only reducible at the topmost position. Since $t_{m} \cdot \gamma$ is not abstracted at the topmost position and $p_{1} \in \operatorname{Border}\left(t_{0} \gamma, \operatorname{Right}\left(R_{E}\right)\right)$, by Lemma $15, t_{i} \gamma \not \equiv t_{0} \gamma \equiv t$ for all $i$ in $\{1, \ldots, m\}$ and, hence, $t_{i} \gamma \not \equiv t$ for all $i$ in $\{k, \ldots, m\}$.

Now, we consider the following, as said, two unique cases where either $s^{\prime} \sigma^{\prime} \equiv$ $t$ or $s^{\prime} \sigma^{\prime} \equiv s$.

(a) $s^{\prime} \sigma^{\prime} \equiv t$. Since $t_{k} \cdot \gamma \curvearrowright^{p_{k+1}} \ldots \curvearrowright^{p_{m}} t_{m} \cdot \gamma$, where $t_{k} \gamma \neq \equiv t$ and, for all $i$ in $\{k+1, \ldots, m\}, p_{i}$ is not below any variable position of $t^{\prime}$ and $t_{i} \gamma \neq \equiv t$, then, by Lemma 9 applied on $s^{\prime} \simeq t^{\prime}$, there is an equation $s^{\prime \prime} \simeq t^{\prime \prime}$ in $E$, where $s^{\prime \prime}$ is subsumed by $s^{\prime}$ and $t^{\prime \prime}[]_{p_{k+1}} \cdots[]_{p_{m}}$ is subsumed by $t^{\prime}[]_{p_{k+1}} \cdots[]_{p_{m}}$, that has an instance of the form $s^{\prime} \sigma^{\prime} \simeq t_{m} \gamma$, i.e., $t \simeq t_{m} \gamma$.

Now, recall that $t \curvearrowright^{*} t_{m} \cdot \gamma$ and $t_{m} \cdot \gamma$ is irreducible by $\curvearrowright$ and thus by $\curvearrowright_{R_{E}^{\circ}}$ except at the topmost position. Therefore, if $t_{m} \cdot \gamma$ is reducible by $\curvearrowright \overline{R_{E}^{\circ}}$ at some position $p$ then either $p=\lambda$ or there is no abstraction below $p$ and, thus, there has been no rewriting step below $p$, which implies that $\left.\left.t_{m} \gamma\right|_{p} \equiv t\right|_{p}$. Moreover, we can show that if $p \notin \operatorname{Border}\left(t, \operatorname{Right}\left(R_{E}\right)\right)$ 
then $p \notin \operatorname{Border}\left(t_{m} \gamma, \operatorname{Right}\left(R_{E}\right)\right)$. Let $q \neq \lambda$ be the closest position to $p$ such that $\left.t\right|_{q} \in \operatorname{Right}\left(R_{E}\right)$. Then we have that $q$ is a non-variable position of $t_{m}$ (as $p$ is) and, since $q \neq \lambda$, we have that $\left.t_{m}\right|_{q} \cdot \gamma$ is normalized (as $t_{m} \cdot \gamma$ is irreducible except at the topmost position). Thus, since $\left.t \succ_{B} t\right|_{q}$, $\left.t\right|_{q}$ is innermost returning by assumption and, if $\left.t_{m}\right|_{q} \cdot \gamma$ is normalized, then $\left.\left.t_{m}\right|_{q} \gamma \equiv t\right|_{q}$ which implies that $p \notin \operatorname{Border}\left(t_{m} \gamma, \operatorname{Right}\left(R_{E}\right)\right)$.

Therefore, the only reducible positions of $t_{m} \gamma$ by $R_{E}^{\circ}$ that belong to $\operatorname{Border}\left(t_{m} \gamma, \operatorname{Right}\left(R_{E}\right)\right)$ are either $\lambda$, or positions $p$ of $t$ with $\left.\left.t_{m} \gamma\right|_{p} \equiv t\right|_{p}$ and $p \in \operatorname{Border}\left(t, \operatorname{Right}\left(R_{E}\right)\right)$. Then, as $R_{E} \subseteq R_{E}^{\circ}$, by Lemma 21 we have that $t_{m} \gamma \succ t$ and, hence, $t_{m} \gamma \rightarrow t$ is a rule in $R_{E}^{\circ}$. Using this rule, we can reduce $t_{m} \cdot \gamma$ obtaining $x \cdot\{x \mapsto t\}$, and we are done with $m=n-1$.

(b) $s^{\prime} \sigma^{\prime} \equiv s$. In this case we have two more possibilities, depending on whether $s^{\prime}$ is a variable or not.

i. If $s^{\prime}$ is not a variable, then $\lambda$ is not below any variable position of $s^{\prime}$ and, obviously, $s \curvearrowright_{\{s \rightarrow t\}}^{\lambda} x \cdot\{x \mapsto t\}$ for some variable $x$. Moreover, as we have seen, we have that $t^{\prime} \sigma^{\prime} \equiv t_{k} \gamma$ and $t_{k} \gamma \not \equiv t$. Therefore, by Lemma 9 applied on $t^{\prime} \simeq s^{\prime}$, there is an equation $t^{\prime \prime} \simeq s^{\prime \prime}$ in $E$, where $t^{\prime \prime}$ is subsumed by $t^{\prime}$, that has an instance of the form $t_{k} \gamma \equiv t^{\prime} \sigma^{\prime} \simeq x\{x \mapsto t\} \equiv t$. Let $\sigma^{\prime \prime}$ be the substitution of that instance. Then we can conclude analogously to case 1 a on $s^{\prime \prime} \simeq t^{\prime \prime}$, since we have $s^{\prime \prime} \sigma^{\prime \prime} \equiv t, t^{\prime \prime} \sigma^{\prime \prime} \equiv t_{k} \gamma$ and all $p_{k+1} \ldots p_{m}$ are not below any variable position of $t^{\prime \prime}$, because it is subsumed by $t^{\prime}$ and they are not below a variable position of $t^{\prime}$.

ii. If $s^{\prime}$ is a variable $x$ then, since $t^{\prime} \sigma \equiv t \not \equiv t_{k} \gamma \equiv t^{\prime} \sigma^{\prime}$ and $s^{\prime} \sigma \equiv s^{\prime} \sigma^{\prime}$, we have that $t^{\prime}$ has some extra variables different from $x$. Let $x^{\prime} \simeq t_{r}^{\prime}$ be a variable renamed version of $x \simeq t^{\prime}$. Now we extend the substitution $\sigma^{\prime}$ with $z^{\prime} \mapsto z \sigma$ if $z^{\prime}$ is the renaming of $z$. Note that now we have $x \sigma^{\prime} \equiv x^{\prime} \sigma^{\prime} \equiv s$ and $t_{r}^{\prime} \sigma^{\prime} \equiv t^{\prime} \sigma \equiv t$, and thus $t_{r}^{\prime} \sigma^{\prime} \not \equiv t^{\prime} \sigma^{\prime}$. Then by Lemma 10, taking $x^{\prime} \simeq t_{r}^{\prime}$ for $l \simeq r, x \simeq t^{\prime}$ for $s \simeq t$ and $\sigma^{\prime}$ for $\sigma$, there is an equation $t_{r}^{\prime \prime} \simeq t^{\prime}$ in $E$, with $t_{r}^{\prime \prime} \sigma^{\prime} \equiv t_{r}^{\prime} \sigma^{\prime} \equiv t$. (Note that, although $s \equiv x \sigma \succ t^{\prime} \sigma \equiv t$, we do not necessarily have $x \sigma^{\prime} \succ t^{\prime} \sigma^{\prime}$.) Again, we can conclude analogously to case 1 a on $t_{r}^{\prime \prime} \simeq t^{\prime}$, since $t_{r}^{\prime \prime} \sigma^{\prime} \equiv t$

2. No step with $\curvearrowright_{\bar{S}}$ has taken place yet at any position in $\operatorname{Border}\left(t, \operatorname{Right}\left(R_{E}\right)\right)$ when we reach $t_{k} \cdot \gamma$ (in particular, it is possible that no step has taken place at all, i.e., we can have that $k=0$ ). In this case, since $S$ is non-overlapping, if $t$ is reducible at some position $p$ by a rule in $S$ then, if $p$ is none of the positions $p_{1}, \ldots, p_{k}$, then $\left.\left.t\right|_{p} \equiv t_{k}\right|_{p}$. Moreover, since $S$ is not empty and every rule in $S$ reduces $t$ at some position in $\operatorname{Border}\left(t, \operatorname{Right}\left(R_{E}\right)\right)$, there is at least one position $p$ in $t_{k} \cdot \gamma$ that can be reduced by $\curvearrowright$ with a rule $l \rightarrow r$ in $S$. Note also that $p \neq \lambda$, since, by Lemma 12 , we have that $\operatorname{Left}\left(R_{E}^{\circ}\right) \cap \operatorname{Right}\left(R_{E}\right)=\emptyset$. Therefore, assuming that $y \gamma \equiv r$, we have that

$$
t_{k} \cdot \gamma \curvearrowright^{p} t_{k}[y]_{p} \cdot \gamma \equiv t_{k+1} \cdot \gamma
$$

Then, similarly to case 1 , let $t_{m} \cdot \gamma$ be an abstracted term obtained in a derivation with $\curvearrowright$ starting with $t_{k+1} \cdot \gamma$, and reducing at all positions but 
$\lambda$. Then, we have

$$
t_{k+1} \cdot \gamma \curvearrowright^{p_{k+2}} t_{k+2} \cdot \gamma \cdots \curvearrowright^{p_{m}} t_{m} \cdot \gamma
$$

and $t_{m} \cdot \gamma$ can only be reducible at the topmost position. Moreover, by confluence, since $t_{0} \cdot \gamma \curvearrowright^{p} t_{0}[y]_{p} \cdot \gamma$, we also have

$$
t_{0} \cdot \gamma \curvearrowright^{p} t_{0}[y]_{p} \cdot \gamma \cdots \curvearrowright t_{k+1} \cdot \gamma \curvearrowright^{p_{k+2}} t_{k+2} \cdot \gamma \cdots \curvearrowright^{p_{m}} t_{m} \cdot \gamma
$$

and, since $t_{m} \cdot \gamma$ is not abstracted at the topmost position and $p$ belongs to $\operatorname{Border}\left(t_{0} \gamma, \operatorname{Right}\left(R_{E}\right)\right)$, by Lemma $15, t_{i} \gamma \not \equiv t_{0} \gamma \equiv t$ for all $i$ in $\{1, \ldots, m\}$ and, hence, $t_{i} \gamma \not \equiv t$ for all $i$ in $\{k+1, \ldots, m\}$. Now, we consider again the two unique cases on $s^{\prime} \sigma^{\prime}$.

(a) $s^{\prime} \sigma^{\prime} \equiv t$. In this case, recall that $t^{\prime} \sigma^{\prime} \equiv t_{k} \gamma$. And $p_{k+1}$ is not below any variable position of $t^{\prime}$. Then, by Lemma 9 applied on $s^{\prime} \simeq t^{\prime}$, there is an equation $s^{\prime \prime} \simeq t^{\prime \prime}$ in $E$, where $s^{\prime \prime}$ is subsumed by $s^{\prime}$ and $t^{\prime \prime}[]_{p_{k+1}}$ is subsumed by $t^{\prime}[]_{p_{k+1}}$, that has an instance of the form $t \simeq t_{k+1} \gamma$. Let $\sigma^{\prime \prime}$ be the substitution of that instance. Then we can conclude analogously to case 1 a on $s^{\prime \prime} \simeq t^{\prime \prime}$ and $\sigma^{\prime \prime}$, but with $k+1$ playing the role of $k$, since $s^{\prime \prime} \sigma^{\prime \prime} \equiv t, t^{\prime \prime} \sigma^{\prime \prime} \equiv t_{k+1} \gamma$ and all $p_{k+2} \ldots p_{m}$ are not below any variable position of $t^{\prime \prime}$, because it is subsumed by $t^{\prime}$ and they are not below a variable position of $t^{\prime}$.

(b) $s^{\prime} \sigma^{\prime} \equiv s$. In this case several new situations, depending mainly on whether a tautology is obtained or not, have to be carefully explored:

i. $t_{k+1} \gamma \not \equiv s$. Then, as in previous cases, by Lemma 9 applied on $s^{\prime} \simeq t^{\prime}$, there is an equation $s^{\prime \prime} \simeq t^{\prime \prime}$ in $E$, where $s^{\prime \prime}$ is subsumed by $s^{\prime}$ and $t^{\prime \prime}[]_{p_{k+1}}$ is subsumed by $t^{\prime}[]_{p_{k+1}}$, that has an instance with $\sigma^{\prime \prime}$ of the form $s \simeq t_{k+1} \gamma$. Now, we can conclude analogously to case $1 \mathrm{~b}$ on $s^{\prime \prime} \simeq t^{\prime \prime}$ and $\sigma^{\prime \prime}$, but with $k+1$ playing the role of $k$, since $s^{\prime \prime} \sigma^{\prime \prime} \equiv s$, $t^{\prime \prime} \sigma^{\prime \prime} \equiv t_{k+1} \gamma$ and all $p_{k+2} \ldots p_{m}$ are not below any variable position of $t^{\prime \prime}$, because it is subsumed by $t^{\prime}$ and they are not below a variable position of $t^{\prime}$.

ii. $t_{k+1} \gamma \equiv s$, and $k>0$, i.e., some steps in $D$ take place below variable positions of $t^{\prime}$. In this case, first of all, we will show that $t_{k} \gamma \not \equiv t$ : Since (i) every rule in $S$ reduces $t$ at $\operatorname{Border}\left(t, \operatorname{Right}\left(R_{E}\right)\right)$, (ii) no step with $\curvearrowright_{\bar{S}}$ has taken place yet at any position in $\operatorname{Border}\left(t, \operatorname{Right}\left(R_{E}\right)\right)$ when we reach $t_{k} \cdot \gamma$, and (iii) $k>0$, then there is at least one rule $l \rightarrow r$ in $S$ reducing $t$ both at some position in $\operatorname{Border}\left(t, \operatorname{Right}\left(R_{E}\right)\right)$ and at some position not in $\operatorname{Border}\left(t, \operatorname{Right}\left(R_{E}\right)\right)$. Therefore, if $t_{k} \gamma \equiv t$, since we use innermost rewriting, after only one rewrite step, at least one occurrence of such redex $l$ would remain in $t_{k+1} \gamma$ at some nontopmost position. But then it would not be possible to have $t_{k+1} \gamma \equiv s$ since, as we have seen, either $l$ does not occur in $s$, or $l \rightarrow r$ and $s \rightarrow t$ are the same rule. Therefore, we have that $t_{k} \gamma \not \equiv t$. Then, we can conclude analogously to case $1 \mathrm{~b}$ on $s^{\prime} \simeq t^{\prime}, \sigma^{\prime}$ and $k$.

iii. $t_{k+1} \gamma \equiv s$, and $k=0$, i.e., no step in $D$ takes place below any variable position of $t^{\prime}$ and, hence, the step at position $p_{k+1}$ is the 
first one. Therefore, for some rule $l \rightarrow r$ in $S$ and some non-variable position $p_{1}$ of $t^{\prime}$, we have that $\left.t\right|_{p_{1}} \equiv l, t_{1} \equiv t\left[x_{1}\right]_{p_{1}}$ for some variable $x_{1}$ and $t_{1} \gamma \equiv t[r]_{p_{1}} \equiv s$.

Now recall that, for every rule $l \rightarrow r$ in $S$, either $l$ does not occur in $s$, or $l \rightarrow r$ and $s \rightarrow t$ are the same rule. Here $s \rightarrow t$ cannot be a rule in $S$ : if this was the case then, as every rule in $S$ reduces $t$ and $s \not \equiv t, s$ would occur in $t$ at some non-topmost position and, hence, also in $t[r]_{p_{1}}$ at some non-topmost position, contradicting $t_{1} \gamma \equiv s$.

Hence, no rule in $S$ reduces $s$. Therefore, since the rules in $S$ are non-overlapping and we have $t[r]_{p_{1}} \equiv s$, it should be the case that $\left.\left.t\right|_{p_{1}} \rightarrow s\right|_{p_{1}}$ is the only rule in $S$, which moreover only reduces $t$ at position $p_{1}$ and $t_{1} \equiv s\left[x_{1}\right]_{p_{1}}$ (note that this orientation of the rule is possible, since monotonicity is not required for the relation $\succ$ ). Note also that in this case $p_{1}$ must be a position in $\operatorname{Border}\left(t, \operatorname{Right}\left(R_{E}\right)\right)$. Now we show that,

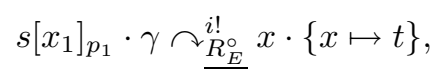

by induction on $\left|s\left[x_{1}\right]_{p_{1}}\right|$.

If $s\left[x_{1}\right]_{p_{1}} \cdot \gamma$ is irreducible by $\curvearrowright$ except at the topmost position, then a last step at the topmost position with $s \rightarrow t$ lets us conclude.

If, otherwise, $s\left[x_{1}\right]_{p_{1}} \cdot \gamma$ is still reducible by $\curvearrowright$ at some position $p_{2} \neq \lambda$, then we have that

$$
t \curvearrowright \frac{p_{1}}{S} s\left[x_{1}\right]_{p_{1}} \cdot \gamma \curvearrowright_{\underline{R_{E}^{\circ}}}^{p_{2}} s\left[x_{2}\right]_{p_{2}} \cdot \gamma,
$$

i.e., we have that $p_{2}$ is strictly above $p_{1}$, since, as stated before, $S$ has a single rule which reduces $t$ only at position $p_{1}$. In this case, let $u \rightarrow v$ be the rule in $R_{E}^{\circ}$ reducing $s\left[x_{1}\right]_{p_{1}} \gamma \equiv s$ at position $p_{2}$. Since $p_{2} \neq \lambda$, we have that $s \simeq t \succ_{e} u \simeq v$ and, hence, by Lemma 8 , $u$ occurs below some righthand side of $R_{E}$ in $s$, i.e., $p_{2}=p \cdot p^{\prime}$ for some $p$ and $p^{\prime}$, where $\left.s\right|_{p} \in \operatorname{Right}\left(R_{E}\right)$. Let $p$ be the closest position to $p_{2}$ such that $\left.s\right|_{p} \in \operatorname{Right}\left(R_{E}\right)$, i.e., that has no strict subterm in $\operatorname{Right}\left(R_{E}\right)$ containing $\left.s\right|_{p_{2}}$. Then, we have that $p^{\prime}$ is in $\operatorname{Border}\left(\left.s\right|_{p}, \operatorname{Right}\left(R_{E}\right)\right)$ and, therefore, we have that $\left.t \succ_{B} s\right|_{p}$, since $s \rightarrow t$ is the rule introducing $t$ in $R_{E},\left.s \triangleright s\right|_{p}$ (note that $p \neq \lambda$ since, by Lemma 11, we have that $\left.\operatorname{Left}\left(R_{E}\right) \cap \operatorname{Right}\left(R_{E}\right)=\emptyset\right)$, and $\left.s\right|_{p}$ is reducible by $R_{E}^{\circ}$ at $\operatorname{Border}\left(\left.s\right|_{p}, \operatorname{Right}\left(R_{E}\right)\right)$. Then, since, by assumption, all smaller terms than $t$ w.r.t. $\succ_{B}$ are innermost returning, we have that

$$
\left.s\right|_{p}\left(\curvearrowright_{\{u \rightarrow v\}} \cup \curvearrowright_{\underline{R_{E}^{\circ}}}\right)^{i !} w \cdot \theta,
$$

with $\left.w \theta \equiv s\right|_{p}$. And, in particular, since $u \rightarrow v$ reduces $\left.s\right|_{p}$ at $\operatorname{Border}\left(\left.s\right|_{p}, \operatorname{Right}\left(R_{E}\right)\right)$, by Lemma 15 we have that $w \equiv y$ for some variable $y$.

Finally, we have that $\left.\left.s\right|_{p} \equiv s\left[x_{1}\right]_{p_{1}}\right|_{p} \gamma$, all non-variable positions of $\left.s\left[x_{1}\right]_{p_{1}}\right|_{p}$ are non-variable positions of $\left.s\right|_{p}$ and, since $\left.s\left[x_{1}\right]_{p_{1}}\right|_{p_{2}} \gamma \equiv u$, 
all reducible positions of $\left.s\right|_{p}$ are reducible positions of $\left.s\left[x_{1}\right]_{p_{1}}\right|_{p} \cdot \gamma$. Therefore, by Lemma 18, we have

$$
\left.s\left[x_{1}\right]_{p_{1}}\right|_{p} \cdot \gamma\left(\curvearrowright_{\{u \rightarrow v\}} \cup \curvearrowright_{\underline{R_{E}^{\circ}}}\right)^{i !} y \cdot \theta,
$$

Using this derivation on $s\left[x_{1}\right]_{p_{1}} \cdot \gamma$ we have

$$
s\left[x_{1}\right]_{p_{1}} \cdot \gamma\left(\curvearrowright_{\{u \rightarrow v\}} \cup \curvearrowright_{\underline{R_{E}^{\circ}}}\right)^{i *} s[y]_{p} \cdot \theta,
$$

and, since $\left|s\left[x_{1}\right]_{p_{1}}\right|>\left|s[y]_{p}\right|$, by the induction hypothesis,

$$
s[y]_{p} \cdot \theta \stackrel{\curvearrowright_{\underline{R_{E}^{\circ}}}^{i !}}{i} \cdot\{x \mapsto t\},
$$

and we are done.

The following Lemma generalizes Lemma 22, in the sense that rules in $S$ are not required to reduce $t$ at the border. With them, we establish the Return Property for the set of righthand sides of $R_{E}$, except that not only rules in $R_{E}$, but rules in $R_{E}^{\circ}$, are used for returning to the original term. Later on we show that, from this result, a result where only rules in $R_{E}$ are used follows.

Lemma 23. Let $E$ be a set of equations that is closed under $\mathcal{E}$. Then all terms in $\operatorname{Right}\left(R_{E}\right)$ are innermost returning.

Proof. We have to prove that for every non-overlapping subset $S$ of $R_{E}^{\circ}$, the normal form $u \cdot \delta$ of $t$ w.r.t. $\left(\curvearrowright_{\bar{S}} \cup \curvearrowright_{R_{E}^{\circ}}\right)^{i}$ fulfills $u \delta \equiv t$. We proceed by induction on $|t|$.

We take the positions of the maximal strict subterms of $t$ belonging to $\operatorname{Right}\left(R_{E}\right)$. Being precise, let $p_{1}, \ldots, p_{n}$ be the set of non-topmost positions of $t$ such that $\left.t\right|_{p_{i}} \in \operatorname{Right}\left(R_{E}\right)$, and $p_{i}$ is not below any other position $q$ of $t$ such that $\left.t\right|_{q} \in \operatorname{Right}\left(R_{E}\right)$.

By the induction hypothesis, $\left.t\right|_{p_{i}}$ is innermost returning for every $i$ and, therefore, for every non-overlapping subset $S$ of $R_{E}^{\circ}$, we have that $\left.t\right|_{p_{i}}\left(\curvearrowright_{\bar{S}} \cup \curvearrowright_{\underline{R_{E}^{\circ}}}\right.$ )$^{i !} u_{i} \cdot \delta_{i}$ with $\left.u_{i} \delta_{i} \equiv t\right|_{p_{i}}$.

Now, consider the abstracted term $t^{\prime} \cdot \delta$, where $\delta=\delta_{1} \cup \ldots \cup \delta_{n}$ and $t^{\prime}=$ $t\left[u_{1}\right]_{p_{1}} \ldots\left[u_{n}\right]_{p_{n}}$. First, we have that $t\left(\curvearrowright_{\bar{S}} \cup \curvearrowright_{R_{E}^{\circ}}\right)^{i *} t^{\prime} \cdot \delta$. Moreover, since $u_{i} \delta_{i} \equiv$ $\left.t\right|_{p_{i}}$ for every $i$ in $1 \ldots m$, we have that $t^{\prime} \delta \equiv t\left[\overline{\left.t\right|_{p_{1}}}\right]_{p_{1}} \ldots\left[\left.t\right|_{p_{n}}\right]_{p_{n}} \equiv t$.

There are two cases to be considered. If $t^{\prime} \cdot \delta$ is irreducible by $\curvearrowright_{\bar{S}} \cup \curvearrowright_{R_{E}^{\circ}}$, then we are done. Otherwise, $t^{\prime} \cdot \delta$ is reducible at $\operatorname{Border}\left(t, \operatorname{Right}\left(R_{E}\right)\right)$, and then we prove that $t^{\prime} \cdot \delta\left(\curvearrowright_{\bar{S}} \cup \curvearrowright_{R_{E}^{\circ}}\right)^{i !} x \cdot\{x \mapsto t\}$ for some variable $x$.

Let $S_{B}$ be the subset of $\bar{S}$ including only those rules reducing $t$ at some position in $\operatorname{Border}\left(t, \operatorname{Right}\left(R_{E}\right)\right)$. Note that $t^{\prime} \cdot \delta$ is reducible by $\curvearrowright_{S}$ if and only if $t^{\prime} \cdot \delta$ is reducible by $\curvearrowright_{\overline{S_{B}}}$, since all positions not at $\operatorname{Border}\left(t, \operatorname{Right}\left(R_{E}\right)\right)$ have already been normalized in $t^{\prime} \cdot \delta$. Moreover, there is no reducible position $p$ of 
$t^{\prime} \cdot \delta$ by $\curvearrowright_{R_{E}^{\circ}}$ such that $\left.t^{\prime}\right|_{p} \delta$ is a subterm of some $l$ in $\operatorname{Left}\left(S_{B}\right)$, since $\left.\left.t^{\prime}\right|_{p} \delta \equiv t\right|_{p}$ must be reducible by $S$ in a proper subterm (as it has been abstracted), $S_{B} \subseteq S$, and all rules in $S$ are non-overlapping.

Therefore, we prove that $t^{\prime} \cdot \delta\left(\curvearrowright_{\overline{S_{B}}} \cup \curvearrowright_{R_{E}^{\circ}}\right)^{i !} x \cdot\{x \mapsto t\}$ for every nonoverlapping subset $S_{B}$ of $R_{E}^{\circ}$ such that there is no position $p$ of $t^{\prime} \cdot \delta$ reducible by $\curvearrowright_{R_{E}^{\circ}}$ where $\left.t^{\prime}\right|_{p} \delta$ is a subterm of some $l$ in $\operatorname{Left}\left(S_{B}\right)$.

We proceed by induction on the number of abstracted positions of $t^{\prime} \cdot \delta$. There are two cases to be considered.

If $t^{\prime} \cdot \delta$ is innermost reducible by $\curvearrowright_{\overline{S_{B}}} \cup \curvearrowright_{R_{E}^{\circ}}$ using a step with $\curvearrowright_{R_{E}^{\circ}}$ at

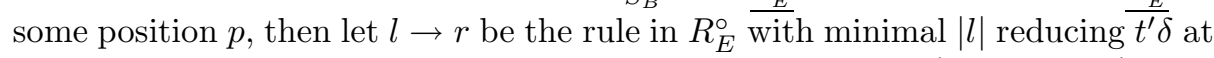
position $p$, and let $q_{1}, \ldots, q_{m}$ be all non-variable positions of $t^{\prime}$ such that $\left.t^{\prime}\right|_{q_{i}} \delta \equiv$ $l$. Note that all $\left.t^{\prime}\right|_{q_{i}} \cdot \delta$ are equivalent and, hence, all such positions are reducible by $\left(\curvearrowright_{R_{E}^{\circ}}\right)^{i}$ with $l \rightarrow r$. Then we have $t^{\prime} \cdot \delta\left(\curvearrowright_{\overline{S_{B}}} \cup \curvearrowright_{R_{E}^{\circ}}\right)^{i+} t^{\prime}[y]_{q_{1}} \ldots[y]_{q_{m}} \cdot \delta^{\prime}$ with $\delta^{\prime}=\delta \bar{\cup}\{y \mapsto r\}$, applying $l \rightarrow r$ in $R_{E}^{\circ}$. In order to show that $t^{\prime}[y]_{q_{1}} \ldots[y]_{q_{m}}$. $\delta^{\prime}\left(\curvearrowright_{\overline{S_{B}}} \cup \curvearrowright_{R_{E}^{\circ}}\right)^{i !} x \cdot\{x \mapsto t\}$, we distinguish two more cases:

- If some rule $l^{\prime} \rightarrow r^{\prime}$ in $S_{B}$ reduces $l$ then, since the step with $l \rightarrow r$ in $R_{E}^{\circ}$ is innermost, $l^{\prime} \rightarrow r^{\prime}$ cannot reduce any $\left.t^{\prime}\right|_{q_{i}} \cdot \delta$ and, hence, $l^{\prime}$ must occur in the abstraction part, i.e., below positions $q$ of $l$ such that $\left.l\right|_{q} \in \operatorname{Right}\left(R_{E}\right)$. Now, by the outer induction, we have that all such $\left.l\right|_{q}$ are innermost returning (since they are strict subterms of $l$ and hence of $t$ ). Therefore, we have that $l\left(\curvearrowright_{\overline{S_{B}}} \cup \curvearrowright_{R_{E}^{\circ}}\right)^{i+} u \cdot \rho$ with $u \rho \equiv l$, by normalizing all subterms of $l$ in $\operatorname{Right}\left(R_{E}\right)$. Note that $u \cdot \rho$ is at most as abstracted as $\left.t^{\prime}\right|_{q_{i}} \cdot \delta$ for all $i$ in $1 \ldots m$, since $S_{B} \subseteq S$ only reduces $l$ at positions that are in the abstracted part of $\left.t^{\prime}\right|_{q_{i}} \cdot \delta$ (recall that if a ground term is irreducible by $\curvearrowright_{\overline{S_{B}}}$ then it is normalized with respect to $\curvearrowright_{\overline{S_{B}}} \cup \curvearrowright_{R_{E}^{\circ}}$ ).

Now, we show that $u \cdot \rho$ is irreducible by $\curvearrowright_{\overline{S_{B}}} \cup \curvearrowright_{R_{E}^{\circ}}$ except at the topmost position, where it can be reduced by $\curvearrowright_{\underline{R_{E}^{\circ}}}$, with the rule $l \rightarrow r$ (since $u \cdot \rho$ has at least one abstracted position). Assume that $u \cdot \rho$ is reducible at some non-topmost position $k$. First, we have that $k$ cannot be below an abstracted position of $\left.t^{\prime}\right|_{q_{i}} \cdot \delta$ since, in that case, by construction, $\left.l\right|_{k}$ has been normalized when obtaining $u \cdot \rho$, and thus it cannot be reducible. Now, let $k$ be a nonabstracted position of $\left.t^{\prime}\right|_{q_{i}} \cdot \delta$. If $u \cdot \rho$ is innermost reducible by $\curvearrowright_{\overline{S_{B}}}$ at position $k$, since $u \cdot \rho$ is at most as abstracted as $\left.t^{\prime}\right|_{q_{i}} \cdot \delta$, then $\left.t^{\prime}\right|_{q_{i}} \cdot \delta$ is either reducible by $\curvearrowright_{\overline{S_{B}}}$ or by $\curvearrowright_{R_{E}^{\circ}}$ at position $k$, contradicting the fact that the step with $l \rightarrow r$ was innermost. If, otherwise, $u \cdot \rho$ is innermost reducible by $\curvearrowright_{R_{E}^{\circ}}$ at position $k$, since $u \cdot \rho$ is at most as abstracted as $\left.t^{\prime}\right|_{q_{i}} \cdot \delta$, then $\left.t^{\prime}\right|_{q_{i}} \cdot \delta$ is reducible by $\curvearrowright_{\underline{R_{E}^{\circ}}}$ at position $k$, leading again to a contradiction.

We can conclude this case, since we have shown that $u \cdot \rho\left(\curvearrowright_{\overline{S_{B}}} \cup \curvearrowright_{R_{E}^{\circ}}\right)^{i} x$. $\{x \mapsto r\}$ using $l \rightarrow r$ in $R_{E}^{\circ}$. Therefore, we have $t^{\prime}[l]_{q_{1}} \ldots[l]_{q_{m}} \cdot \delta\left(\curvearrowright_{\overline{S_{B}}} \overline{\cup \curvearrowright_{R_{E}}^{\circ}}\right.$ )$^{i+} t^{\prime}[y]_{q_{1}} \ldots[y]_{q_{m}} \cdot \delta^{\prime}$ and, since $t^{\prime}[l]_{q_{1}} \ldots[l]_{q_{m}} \cdot \delta$ has less abstracted positions 
than $t^{\prime} \cdot \delta$, by the induction hypothesis, $t^{\prime}[l]_{q_{1}} \ldots[l]_{q_{m}} \cdot \delta\left(\curvearrowright_{\overline{S_{B}}} \cup \curvearrowright_{R_{E}^{\circ}}\right)^{i !} x$. $\{x \mapsto t\}$. Then, by confluence, we have $t^{\prime}[y]_{q_{1}} \ldots[y]_{q_{m}} \cdot \delta^{\prime}\left(\curvearrowright_{\overline{S_{B}}} \bar{\cup} \curvearrowright_{\underline{R_{E}^{\circ}}}\right.$ )$^{i !} x \cdot\{x \mapsto t\}$, and we are done.

- If no rule in $S_{B}$ reduces $l$, then let $S_{B}^{\prime}=S_{B} \cup\{l \rightarrow r\}$. We have that $t^{\prime}[l]_{q_{1}} \ldots[l]_{q_{m}} \cdot \delta\left(\curvearrowright_{\overline{S_{B}^{\prime}}} \cup \curvearrowright_{R_{E}^{\circ}}\right)^{i+} t^{\prime}[y]_{q_{1}} \ldots[y]_{q_{m}} \cdot \delta^{\prime}$, applying $l \rightarrow r$ in $S_{B}^{\prime}$. By minimality of $|l|$, no step with $\curvearrowright_{R_{E}^{\circ}}$ can be done on $t^{\prime}[l]_{q_{1}} \ldots[l]_{q_{m}} \cdot \delta$ with a rule reducing $l$. Hence, $S_{B}^{\prime}$ fulfills the conditions imposed on $S_{B}$, and $t^{\prime}[l]_{q_{1}} \ldots[l]_{q_{m}} \cdot \delta$ has less abstracted positions than $t^{\prime} \cdot \delta$ and, hence, by the induction hypothesis, $t^{\prime}[l]_{q_{1}} \ldots[l]_{q_{m}} \cdot \delta\left(\curvearrowright_{\overline{S_{B}^{\prime}}} \cup \curvearrowright_{R_{E}^{\circ}}\right)^{i !} x \cdot\{x \mapsto t\}$. Now, by confluence, since $\left.t^{\prime}[l]_{q_{1}} \ldots[l]_{q_{m}} \cdot \delta\left(\curvearrowright_{\overline{S_{B}^{\prime}}} \cup \curvearrowright_{R_{E}^{\circ}}\right)^{i+} \overline{t^{\prime}[y}\right]_{q_{1}} \ldots[y]_{q_{m}} \cdot \delta^{\prime}$ and $t^{\prime}[l]_{q_{1}} \ldots[l]_{q_{m}} \cdot \delta\left(\curvearrowright_{\overline{S_{B}^{\prime}}} \cup \curvearrowright_{R_{E}^{\circ}}\right)^{i !} x \cdot\{x \mapsto t\}$, we have that $t^{\prime}[y]_{q_{1}} \ldots[y]_{q_{m}}$. $\delta^{\prime}\left(\curvearrowright_{\overline{S_{B}^{\prime}}} \cup \curvearrowright_{\underline{R_{E}^{\circ}}}\right) i ! x \cdot\{x \mapsto \overline{t\}}$. Finally, since $l \rightarrow r$ cannot be further applied as a rule of $S_{B}^{\prime}$ in any derivation with $\left(\curvearrowright_{\overline{S_{B}^{\prime}}} \cup \curvearrowright_{\underline{R_{E}^{\circ}}}\right)$ starting from $t^{\prime}[y]_{q_{1}} \ldots[y]_{q_{m}} \cdot \delta^{\prime}$, we can conclude that $t^{\prime}[y]_{q_{1}} \ldots[y]_{q_{m}} \cdot \overline{\delta^{\prime}}\left(\curvearrowright_{\overline{S_{B}}} \cup \curvearrowright_{\underline{R_{E}^{\circ}}}\right.$ )$^{i !} x \cdot\{x \mapsto t\}$, and we are done.

We are left with the case where $t^{\prime} \cdot \delta$ is only innermost reducible by $\curvearrowright \overline{S_{B}}$ $\cup \curvearrowright_{R_{E}^{\circ}}$ using steps with $\curvearrowright_{\overline{S_{B}}}$. Let $a_{1}, \ldots, a_{j}$ be all the abstracted positions of $t^{\prime} \cdot \delta$ and let $z_{1}, \ldots, z_{j}$ be the variables at those positions. Then every $\left.z_{i} \delta \equiv t\right|_{a_{i}}$ is a term in $\operatorname{Right}\left(R_{E}\right)$ and since, by the induction hypothesis, it is innermost returning, we have that $\left.t\right|_{a_{i}}\left(\curvearrowright_{\overline{S_{B}}} \cup \curvearrowright_{R_{E}^{\circ}}\right)^{i !} w_{i} \cdot \rho_{i}$ with $\left.t\right|_{a_{i}} \equiv w_{i} \rho_{i}$. Therefore, taking $\rho=\rho_{1} \cup \ldots \cup \rho_{j}$, we have that

$$
t\left(\curvearrowright_{\overline{S_{B}}} \cup \curvearrowright_{\underline{R_{E}^{\circ}}}\right)^{i *} t\left[w_{1}\right]_{a_{1}} \ldots\left[w_{j}\right]_{a_{j}} \cdot \rho .
$$

On the other hand, by Lemma 22, we have that

$$
t\left(\curvearrowright_{\overline{S_{B}}} \cup \curvearrowright_{\underline{R_{E}^{\circ}}}\right)^{i !} x \cdot\{x \mapsto t\} .
$$

Therefore, by confluence, we have that

$$
t\left[w_{1}\right]_{a_{1}} \ldots\left[w_{j}\right]_{a_{j}} \cdot \rho\left(\curvearrowright_{\overline{S_{B}}} \cup \curvearrowright_{\underline{R_{E}^{\circ}}}\right)^{i !} x \cdot\{x \mapsto t\} .
$$

Since in $t\left[w_{1}\right]_{a_{1}} \ldots\left[w_{j}\right]_{a_{j}} \cdot \rho$ we only have normalized the abstracted positions of $t^{\prime} \cdot \delta$, we have that all non-variable positions of $t^{\prime}$ are non-variable positions of $t\left[w_{1}\right]_{a_{1}} \ldots\left[w_{j}\right]_{a_{j}}$. Additionally, since since $t\left[w_{1}\right]_{a_{1}} \ldots\left[w_{j}\right]_{a_{j}} \rho \equiv t^{\prime} \delta$ and $t^{\prime} \cdot \delta$ is only innermost reducible by $\curvearrowright_{\overline{S_{B}}} \cup \curvearrowright_{R_{E}^{\circ}}$ using steps with $\curvearrowright_{\overline{S_{B}}}$, we have that $t\left[w_{1}\right]_{a_{1}} \ldots\left[w_{j}\right]_{a_{j}} \cdot \rho$ is also only innermost reducible by $\curvearrowright_{\overline{S_{B}}} \cup \curvearrowright_{R_{E}^{\circ}}$ using steps with $\curvearrowright_{\overline{S_{B}}}$ at the same positions.

Then, by Lemma 18, we can conclude that $t^{\prime} \cdot \delta\left(\curvearrowright_{\overline{S_{B}}} \cup \curvearrowright_{R_{E}^{\circ}}\right)^{i !} x \cdot\{x \mapsto t\}$.

Now, roughly, we have to go from the Return Property with $R_{E}^{\circ}$ to the Return Property with $R_{E}$. To this end we show that, for some particular abstracted 
terms, the so-called innermost reduced terms, innermost $B$-rewrite sequences with $R_{E}^{\circ}$ are in fact $B$-rewrite sequences with $R_{E}$.

Definition 15 (Innermost reduced term). Let $R$ be a ground TRS and $s \cdot \gamma$ be an abstracted term. We say that $s \cdot \gamma$ is innermost reduced for $R$ if there is no reducible position $p$ of $s \cdot \gamma$ by $\curvearrowright_{R}$ such that $\operatorname{Var}\left(\left.s\right|_{p}\right)=\emptyset$ (i.e., there is no redex in $s \cdot \gamma$ which has never been reduced inside).

Lemma 24. Let $R$ be a ground TRS, and $s \cdot \gamma$ be an abstracted term which is innermost reduced for $R$. If $s \cdot \gamma \curvearrowright_{R} t \cdot \delta$, then $t \cdot \delta$ is innermost reduced for $R$.

Proof. Let $t \cdot \delta$ be of the form $s[x]_{p} \cdot(\gamma \cup\{x \mapsto r\})$ for some rule $l \rightarrow r$ in $R$. On the one hand, we have that $\operatorname{Var}\left(\left.s\right|_{p^{\prime}}\right) \neq \emptyset$ for every prefix $p^{\prime}$ of $p$. On the other hand, for every position $q$ which is not overlapped with $p$ we have that $\operatorname{Var}\left(\left.s\right|_{q}\right)=\operatorname{Var}\left(\left.s[x]_{p}\right|_{q}\right)$, since $\left.\left.s\right|_{q} \equiv s[x]_{p}\right|_{q}$. Therefore, since $s \cdot \gamma$ is innermost reduced for $R$ and, by definition of $B$-rewriting, only positions strictly above $p$ or positions which are not overlapped with $p$ can be $B$-reducible, we have that $t \cdot \delta$ is innermost reduced for $R$.

The following lemma states which subset of rules of $R_{E}^{\circ}$ corresponds to $R_{E}$.

Lemma 25. Let $E$ be a set of equations that is closed under $\mathcal{E}$, and $s \rightarrow t$ a rule in $R_{E}^{\circ}$ such that $s$ is irreducible by $R_{E}$ at every non-topmost position belonging to StrictContext $\left(s, \operatorname{Right}\left(R_{E}\right)\right)$. Then $s \rightarrow t$ belongs to $R_{E}$.

Proof. Assume the contrary, i.e., that such a rule $s \rightarrow t$ is not a rule in $R_{E}$. Then, by Lemma 5 , we have that $s$ is reducible by $R_{E}^{s \simeq t}$ at some position $p$ in StrictContext $\left(s, \operatorname{Right}\left(R_{E}^{s \simeq t}\right)\right)$ and, by Lemma 3, we have that $p$ belongs to StrictContext $\left(s, \operatorname{Right}\left(R_{E}\right)\right)$. Since $R_{E}^{s \simeq t} \subseteq R_{E}$, by assumption, $s$ can only be reducible at position $\lambda$, by some rule $s \rightarrow u$ in $R_{E}$. However, by Lemma 14, there are no two rules in $R_{E}^{\circ}$ with the same lefthand side, which, since $R_{E} \subseteq R_{E}^{\circ}$, implies that $s \rightarrow u$ and $s \rightarrow t$ are the same rule and hence $s \rightarrow t \in R_{E}$.

Lemma 26. Let $E$ be a set of equations that is closed under $\mathcal{E}$, and $s \cdot \gamma$ be an abstracted term w.r.t. Right $\left(R_{E}\right)$ which is innermost reduced for $R_{E}^{\circ}$. Then any rewrite sequence of the form

$$
s \cdot \gamma \curvearrowright_{\underline{R_{E}^{\circ}}}^{i *} t \cdot \delta
$$

is a rewrite sequence with $\curvearrowright_{R_{E}}^{i}$.

Proof. We proceed by induction on the length of the derivation. If there are no steps, we are done. Otherwise, let $s \cdot \gamma \curvearrowright_{R_{E}^{\circ}}^{i} s[x]_{p} \cdot(\gamma \cup\{x \mapsto r\})$ be the first step in the derivation, where $\left.s\right|_{p} \gamma \rightarrow r$ is a rule in $R_{E}^{\circ}$.

Since the rewrite sequence is innermost and $s \cdot \gamma$ is innermost reduced for $R_{E}^{\circ}$ then, apart from $\lambda,\left.s\right|_{p} \gamma$ can be reducible by $R_{E}^{\circ}$ at most below blocked positions of $\left.s\right|_{p} \cdot \gamma$. Now note that, since $s \cdot \gamma$ is abstracted w.r.t. $\operatorname{Right}\left(R_{E}\right)$, then in all blocked positions of $\left.s\right|_{p} \cdot \gamma$ there are subterms belonging to $\operatorname{Right}\left(R_{E}\right)$. Therefore 
we have that $\left.s\right|_{p} \gamma$ is irreducible by $R_{E}^{\circ}$, and hence by $R_{E}$, at every non-topmost position in StrictContext $\left(\left.s\right|_{p} \gamma, \operatorname{Right}\left(R_{E}\right)\right)$.

Then, by Lemma 25, we have that $\left.s\right|_{p} \gamma \rightarrow r \in R_{E}$. Moreover, since by Lemma 24 we have that $s[x]_{p} \cdot(\gamma \cup\{x \mapsto r\})$ is innermost reduced for $R_{E}^{\circ}$ and it is abstracted w.r.t. $\operatorname{Right}\left(R_{E}\right)$, by the induction hypothesis, we have that $s[x]_{p} \cdot(\gamma \cup\{x \mapsto r\}) \curvearrowright_{\underline{R_{E}}}^{i *} t \cdot \delta$ is a sequence with $\curvearrowright_{R_{E}}^{i}$.

The following lemma states that $B$-rewrite derivations are preserved if we have less blocked positions.

Lemma 27. Let $R$ be a TRS and $t \cdot \gamma$ be an abstracted term. If $t \cdot \gamma \curvearrowright_{R}^{+} x \cdot\{x \mapsto$ $t \gamma\}$ with $n$ non-abstraction steps then $t^{\prime} \cdot \gamma^{\prime} \curvearrowright_{R}^{+} x \cdot\{x \mapsto t \gamma\}$ with the same $n$ steps for every abstraction $t^{\prime} \cdot \gamma^{\prime}$ of $t \gamma$ such that all non-variable positions of $t$ are non-variable positions of $t^{\prime}$.

Proof. We proceed by induction on $n$. If $n=1$ then there is only a step at position $\lambda$ and since, by assumption, $t^{\prime} \gamma^{\prime} \equiv t \gamma$ and $t^{\prime} \cdot \gamma^{\prime}$ cannot be abstracted at the topmost position, we have that $t^{\prime} \cdot \gamma^{\prime} \curvearrowright_{R}^{\lambda} x \cdot\{x \mapsto t \gamma\}$ with the same step.

If $n>1$ then we have that $t \cdot \gamma \curvearrowright_{R}^{p_{1}} t[y]_{p_{1}} \cdot(\gamma \cup\{y \mapsto r\}) \equiv t_{1} \cdot \gamma_{1}$ with some rule $l \rightarrow r$ such that $\left.t\right|_{p_{1}} \gamma \equiv l$. Moreover, again by assumption, $\left.t^{\prime}\right|_{p_{1}} \gamma^{\prime} \equiv l$ and, hence, we have that $t^{\prime} \cdot \gamma^{\prime} \curvearrowright_{R}^{p_{1}} t^{\prime}[y]_{p_{1}} \cdot\left(\gamma^{\prime} \cup\{y \mapsto r\}\right) \equiv t_{1}^{\prime} \cdot \gamma_{1}^{\prime}$. We also have that $t_{1}^{\prime} \cdot \gamma_{1}^{\prime}$ is an abstraction of $t_{1} \gamma_{1}$, and all non-variable positions of $t_{1}^{\prime}$ are non-variable positions of $t_{1}$. Then, since $t_{1} \cdot \gamma_{1} \curvearrowright_{R}^{+} x \cdot\{x \mapsto t \gamma\}$ with $n-1$ steps, by the induction hypothesis, we have that $t_{1}^{\prime} \cdot \gamma_{1}^{\prime} \curvearrowright_{R}^{+} x \cdot\{x \mapsto t \gamma\}$ with the same $n-1$ steps.

Now we are ready to obtain the Return Property for rewrite sequences using $R_{E}$, by carefully choosing a subset $S$ of $R_{E}$ and combining lemmas 23 and 26 .

Theorem 1 (Return Property). Let $E$ be a set of equations that is closed under $\mathcal{E}$, and $t \cdot \gamma$ be an abstraction w.r.t. Right $\left(R_{E}\right)$ of a term in $\operatorname{Right}\left(R_{E}\right)$. If $t \cdot \gamma \curvearrowright_{R_{E}} u \cdot \delta$ then $u \cdot \delta \curvearrowright_{R_{E}}^{*} x \cdot\{x \mapsto t \gamma\}$ for some variable $x$.

Proof. We proceed by induction on the size of $t \gamma$. If the step from $t \cdot \gamma$ to $u \cdot \delta$ is an abstraction step, then we have that $u \delta \equiv t \gamma$ and, hence, in the case that $u \cdot \delta$ is not already abstracted at the topmost position, by a last abstraction step at the topmost position we can get $x \cdot\{x \mapsto t \gamma\}$ for some variable $x$.

If, otherwise, we do not have an abstraction step, then $u \cdot \delta$ is of the form $t[x]_{p} \cdot(\gamma \cup\{x \mapsto r\})$, where $\left.t\right|_{p} \gamma \rightarrow r$ is a rule in $R_{E}$.

Now, if $p$ is below some position $p^{\prime}$ such that $\left.\right|_{p^{\prime}} \gamma \in \operatorname{Right}\left(R_{E}\right)$ and $p^{\prime} \neq \lambda$, then, by the induction hypothesis and monotonicity of $B$-rewriting, we have that $t[x]_{p} \cdot(\gamma \cup\{x \mapsto r\}) \curvearrowright_{R_{E}}^{*} t[y]_{p^{\prime}} \cdot\left(\gamma \cup\left\{\left.y \mapsto t\right|_{p^{\prime}} \gamma\right\}\right)$ for some variable $y$ and, hence, by a last abstraction step at the topmost position, we are done.

If $p$ is in $\operatorname{Border}\left(t \gamma, \operatorname{Right}\left(R_{E}\right)\right)$, then let

$$
S=\left\{l \rightarrow r \in R_{E} \mid l \rightarrow r \text { reduces } t \gamma \text { and } l \text { is minimal w.r.t. } \triangleright\right\} .
$$


Note that the rule $\left.t\right|_{p} \gamma \rightarrow r$ does not necessarily belong to $S$. But, in any case, we have that $S$ is non-overlapping, by minimality of its lefthand sides w.r.t. $\triangleright$ and Lemma 13. Then, by Lemma 23, we have

$$
t \gamma\left(\curvearrowright_{S} \cup \curvearrowright_{\underline{R_{E}^{\circ}}}\right) i ! \sigma,
$$

where $v \sigma \equiv t \gamma$. On the other hand, by Lemma 23, applied to all maximal strict subterms of $t \gamma$ in $\operatorname{Right}\left(R_{E}\right)$, we have

$$
t \gamma\left(\curvearrowright_{S} \cup \curvearrowright_{\underline{R_{E}^{\circ}}}\right)^{i+} u \cdot \sigma,
$$

where $u \sigma \equiv t \gamma$ and $u \cdot \sigma$ is only reducible at positions in $\operatorname{Border}\left(t \gamma, \operatorname{Right}\left(R_{E}\right)\right)$.

Now, by confluence, we have

$$
u \cdot \sigma\left(\curvearrowright_{\bar{S}} \cup \curvearrowright_{\underline{R_{E}^{\circ}}}\right)^{i !} v \cdot \sigma
$$

and, moreover, $u \cdot \sigma$ is innermost reducible at position $p$ by $\curvearrowright_{\bar{S}} \cup \curvearrowright_{R_{E}^{\circ}}$ using $\left.t\right|_{p} \gamma \rightarrow r$, since (i) $p$ is a non-variable position of $u$, because it is a position in $\operatorname{Border}\left(t \gamma, \operatorname{Right}\left(R_{E}\right)\right)$ and we have only reduced $t \gamma$ at positions not in $\operatorname{Border}\left(t \gamma, \operatorname{Right}\left(R_{E}\right)\right)$, and (ii) it is innermost because $\left.t\right|_{p} \gamma \rightarrow r \in R_{E}$, by Lemma 7 we have that $\left.t\right|_{p} \gamma$ can only be reduced in positions not in the $\operatorname{Border}\left(\left.t\right|_{p} \gamma, \operatorname{Right}\left(R_{E}\right)\right)$ and $u \cdot \sigma$ is only reducible at positions belonging to $\operatorname{Border}\left(t \gamma, \operatorname{Right}\left(R_{E}\right)\right)$.

Since $u \cdot \sigma$ is innermost reducible at position $p$ with the rule $\left.t\right|_{p} \gamma \rightarrow r$, by confluence we have

$$
u \cdot \sigma\left(\curvearrowright_{\bar{S}} \cup \curvearrowright_{\underline{R_{E}^{\circ}}}\right)^{i} u[y]_{p} \cdot(\sigma \cup\{y \mapsto r\})\left(\curvearrowright_{\bar{S}} \cup \curvearrowright_{\underline{R_{E}^{\circ}}}\right)^{i !} v \cdot \sigma,
$$

and since $p \in \operatorname{Border}\left(t \gamma, \operatorname{Right}\left(R_{E}\right)\right)$, by Lemma $15, v \cdot \sigma \equiv x \cdot\{x \mapsto t \gamma\}$.

Let $t^{\prime} \cdot \gamma^{\prime}$ be $u \cdot \sigma$ after abstracting the non-variable positions of $u$ that are variable positions of $t$. Since $u \cdot \sigma$ is only reducible at positions belonging to $\operatorname{Border}\left(t \gamma, \operatorname{Right}\left(R_{E}\right)\right)$, we have only abstracted irreducible terms, and hence all reducible positions of $u \cdot \sigma$ by $\left(\curvearrowright_{\bar{S}} \cup \curvearrowright_{R_{E}^{\circ}}\right)^{i}$ are reducible positions of $t^{\prime} \cdot \gamma^{\prime}$ by $\left(\curvearrowright_{\bar{S}} \cup \curvearrowright_{\underline{R_{E}^{\circ}}}\right)^{i}$. Then, by Lemma 18 ,

$$
t^{\prime} \cdot \gamma^{\prime}\left(\curvearrowright_{\bar{S}} \cup \curvearrowright_{\underline{R_{E}^{\circ}}}\right)^{i} t^{\prime}[y]_{p} \cdot\left(\gamma^{\prime} \cup\{y \mapsto r\}\right)\left(\curvearrowright_{\bar{S}} \cup \curvearrowright_{\underline{R_{E}^{\circ}}}\right)^{i !} x \cdot\{x \mapsto t \gamma\}
$$

and, by innermost confluence, applying first the steps with $\curvearrowright_{\bar{S}}$, there is some $w \cdot \rho$ such that

$$
t^{\prime} \cdot \gamma^{\prime} \curvearrowright_{\frac{i *}{S}} w \cdot \rho\left(\curvearrowright_{\bar{S}} \cup \curvearrowright_{\underline{R_{E}^{\circ}}}\right)^{i} w[y]_{p} \cdot(\rho \cup\{y \mapsto r\})\left(\curvearrowright_{\underline{R_{E}^{\circ}}}\right)^{i !} x \cdot\{x \mapsto t \gamma\} .
$$

Now, we show that $w[y]_{p} \cdot(\rho \cup\{y \mapsto r\})$ is innermost reduced for $R_{E}^{\circ}$. Assume that there is some non-variable position $q$ of $w[y]_{p}$ reducible by $R_{E}^{\circ}$ with no variable position below it. Let $w^{\prime}$ be the subterm at position $q$. Then, since $t^{\prime} \gamma^{\prime} \equiv t \gamma$ and no step has been applied below $q$ we have that $w^{\prime}$ is a subterm of $t \gamma$, reducible 
by $R_{E}^{\circ}$ and not by $S$, which leads to a contradiction: by Lemma 25 all rules in $R_{E}^{\circ}$ are either in $R_{E}$ or are reducible by some rule in $R_{E}$ and hence, by definition of $S$, all rules in $R_{E}^{\circ}$ reducing $t \gamma$ are in $S$ or are reducible by $S$.

Now, by Lemma 26 and $S \subseteq R_{E}$,

$$
t^{\prime} \cdot \gamma^{\prime} \curvearrowright_{R_{E}}^{i *} w \cdot \rho \curvearrowright_{R_{E}}^{i} w[y]_{p} \cdot(\rho \cup\{y \mapsto r\}) \curvearrowright_{R_{E}}^{i *} x \cdot\{x \mapsto t \gamma\}
$$

and, again by innermost confluence, applying first the step on $p$, we have

$$
t^{\prime} \cdot \gamma^{\prime} \curvearrowright_{R_{E}}^{i} t^{\prime}[y]_{p} \cdot\left(\gamma^{\prime} \cup\{y \mapsto r\}\right) \curvearrowright_{R_{E}}^{i+} x \cdot\{x \mapsto t \gamma\}
$$

and finally, by Lemma 27, removing the necessary abstractions we have

$$
t[y]_{p} \cdot(\gamma \cup\{y \mapsto r\}) \curvearrowright_{R_{E}}^{+} x \cdot\{x \mapsto t \gamma\}
$$

\subsection{Rewrite proofs for the equational case}

In order to prove that we have $B$-rewrite proofs for all consequences of a closed set of equations $E$, we first need to prove the confluence of the rewrite relation $\curvearrowright_{R_{E}}^{+}$on abstracted terms.

The Return Property is the cornerstone for proving local confluence. By construction of $R_{E}$,

- lefthand sides of $R_{E}$ can only overlap under terms that correspond to righthand sides of $R_{E}$ (Lemma 7 ), and

- righthand sides of $R_{E}$ fulfill the Return Property (Theorem 1).

Hence, using the Return Property we can show that, if we reduce an abstraction of a lefthand side of a rule in $R_{E}$ (not at top position), then there is a $B$-rewrite sequence going back to (another abstraction of) the lefthand side again. So the diamond closes with a final step with the same rule.

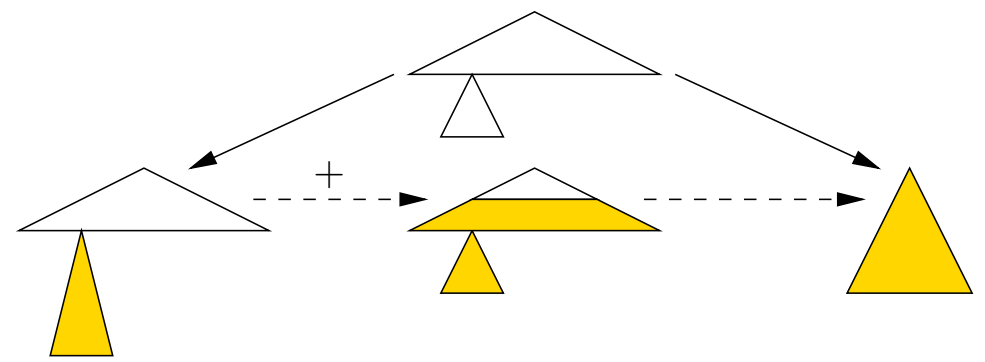

Figure 4. Graphical proof of local confluence. 
Lemma 28 (Local confluence). Let $E$ be a set of equations that is closed under $E$, and $s \cdot \gamma$ be an abstracted term w.r.t. Right $\left(R_{E}\right)$. If $t \cdot \gamma_{1} \curvearrowleft_{R_{E}} s \cdot \gamma \curvearrowright_{R_{E}}$ $u \cdot \gamma_{2}$, then $t \cdot \gamma_{1} \curvearrowright_{R_{E}}^{*} v \cdot \delta \curvearrowleft_{R_{E}}^{*} u \cdot \gamma_{2}$ for some abstracted term $v \cdot \delta$.

Proof. If $t \cdot \gamma_{1} \curvearrowleft_{R_{E}} s \cdot \gamma \curvearrowright_{R_{E}} u \cdot \gamma_{2}$, we have that $t \equiv s[x]_{p_{1}}$ for some fresh variable $x$ and position $p_{1}$, and $\gamma_{1}=\gamma \cup\left\{x \mapsto r_{1}\right\}$, where $\left.s \gamma\right|_{p_{1}} \rightarrow r_{1} \in R_{E}$ or $\left.s \gamma\right|_{p_{1}} \equiv r_{1} \in \operatorname{Right}\left(R_{E}\right)$; and $u \equiv s[y]_{p_{2}}$ for some fresh variable $y$ and position $p_{2}$, and $\gamma_{2}=\gamma \cup\left\{y \mapsto r_{2}\right\}$, where $\left.s \gamma\right|_{p_{2}} \rightarrow r_{2} \in R_{E}$ or $\left.s \gamma\right|_{p_{2}} \equiv r_{2} \in \operatorname{Right}\left(R_{E}\right)$. The following cases have to be considered:

1. There is no overlapping between $p_{1}$ and $p_{2}$. In this case, we can easily close the diamond by applying either $\left.s \gamma\right|_{p_{2}} \rightarrow r_{2}$ or an abstraction step at position $p_{2}$ in $t \cdot \gamma_{1}$, and either $\left.s \gamma\right|_{p_{1}} \rightarrow r_{1}$ or an abstraction step at position $p_{1}$ in $u \cdot \gamma_{2}$, obtaining $v=s[x]_{p_{1}}[y]_{p_{2}}$ and $\delta=\gamma \cup\left\{x \mapsto r_{1}\right\} \cup\left\{y \mapsto r_{2}\right\}$.

2. There is an overlapping between $p_{1}$ and $p_{2}$. In this case assume, w.l.o.g. that $p_{2}$ is below $p_{1}$, and hence $p_{2}=p_{1} \cdot p_{3}$ for some $p_{3}$. Since, by Lemmas 11 and 13 , lefthand sides of rules in $R_{E}$ are not righthand sides and moreover they are unique, we assume that $p_{3} \neq \lambda$ (otherwise we have either two abstraction steps which are the same or two steps with the same rule at the same position, and hence the diamond closes trivially).

Then, since the relation $\curvearrowright_{R_{E}}$ is monotonic, it is enough to show that we can close the diamond starting from $\left.s\right|_{p_{1}} \cdot \gamma$, i.e., we have to show that there is some abstracted term $v^{\prime} \cdot \delta^{\prime}$ such that $x \cdot\left\{x \mapsto r_{1}\right\} \curvearrowright_{R_{E}}^{*} v^{\prime} \cdot \delta^{\prime} \curvearrowleft_{R_{E}}^{*}$ $\left.s\right|_{p_{1}}[y]_{p_{3}} \cdot\left(\gamma \cup\left\{y \mapsto r_{2}\right\}\right)$. The following cases have to be considered:

(a) Both steps are abstraction steps, i.e., $\left.s \gamma\right|_{p_{1}} \equiv r_{1} \in \operatorname{Right}\left(R_{E}\right)$ and $\left.s \gamma\right|_{p_{2}} \equiv r_{2} \in \operatorname{Right}\left(R_{E}\right)$. Then we can apply an abstraction step at the topmost position in $\left.s\right|_{p_{1}}[y]_{p_{3}} \cdot\left(\gamma \cup\left\{y \mapsto r_{2}\right\}\right)$ obtaining $x \cdot\left\{x \mapsto r_{1}\right\}$. Hence, taking $v^{\prime}=x$ and $\delta^{\prime}=\left\{x \mapsto r_{1}\right\}$, it holds.

(b) Only the lower step is an abstraction, i.e., $\left.s \gamma\right|_{p_{1}} \rightarrow r_{1} \in R_{E}$ and $\left.s \gamma\right|_{p_{2}} \equiv$ $r_{2} \in \operatorname{Right}\left(R_{E}\right)$. In this case, we can apply $\left.s \gamma\right|_{p_{1}} \rightarrow r_{1}$ on $\left.s\right|_{p_{1}}[y]_{p_{3}} \cdot(\gamma \cup$ $\left\{y \mapsto r_{2}\right\}$ ), and we conclude as in the previous case.

(c) Only the upper step is an abstraction, i.e., $\left.s \gamma\right|_{p_{1}} \equiv r_{1} \in \operatorname{Right}\left(R_{E}\right)$ and $\left.s \gamma\right|_{p_{2}} \rightarrow r_{2} \in R_{E}$. Then, by Theorem 1, we have that

$$
\left.s\right|_{p_{1}}[y]_{p_{3}} \cdot\left(\gamma \cup\left\{y \mapsto r_{2}\right\}\right) \curvearrowright_{R_{E}}^{*} x \cdot\left\{\left.x \mapsto s \gamma\right|_{p_{1}}\right\},
$$

and we conclude as in the previous cases.

(d) Neither step is an abstraction, i.e., $\left.s \gamma\right|_{p_{1}} \rightarrow r_{1} \in R_{E}$ and $\left.s \gamma\right|_{p_{2}} \rightarrow r_{2} \in$ $R_{E}$. Then, by Lemma 7, there is some position $p_{4}$ above $p_{3}$ in $\left.s \gamma\right|_{p_{1}}$ such that $\left.s \gamma\right|_{p_{1} \cdot p_{4}} \in \operatorname{Right}\left(R_{E}\right)$. Then, by Theorem 1 , we have that

$$
\left.s\right|_{p_{1} \cdot p_{4}}[y]_{p_{5}} \cdot\left(\gamma \cup\left\{y \mapsto r_{2}\right\}\right) \curvearrowright_{R_{E}}^{*} z \cdot\left\{\left.z \mapsto s \gamma\right|_{p_{1} \cdot p_{4}}\right\}
$$

for some variable $z$, where $p_{3}=p_{4} \cdot p_{5}$. Moreover since, by Lemma 11, $\operatorname{Left}\left(R_{E}\right) \cap \operatorname{Right}\left(R_{E}\right)=\emptyset$, we have that $p_{4} \neq \lambda$. Therefore, applying finally $\left.s \gamma\right|_{p_{1}} \rightarrow r_{1}$ on $s_{p_{1}}[z]_{p_{4}} \cdot\left\{\left.z \mapsto s \gamma\right|_{p_{1} \cdot p_{4}}\right\}$, we have that

$$
\left.x \cdot\left\{x \mapsto r_{1}\right\} \curvearrowleft_{R_{E}}^{*} s\right|_{p_{1}}[y]_{p_{3}} \cdot\left(\gamma \cup\left\{y \mapsto r_{2}\right\}\right),
$$

which implies that, taking $v^{\prime}=x$ and $\delta^{\prime}=\left\{x \mapsto r_{1}\right\}$, it holds. 
Since $\curvearrowright_{R_{E}}$ is terminating and locally confluent, it is confluent.

Theorem 2 (Confluence). Let $E$ be a set of equations that is closed under $\mathcal{E}$. Then $\curvearrowright_{R_{E}}$ is confluent.

Now we prove that all equations in $E$ have a $B$-rewrite proof using $R_{E}$, which also implies that $R_{E}^{*}$ is a model for $E$ : first, we show that there is a $B$-rewrite proof for all instances which are irreducible (at positions which are not below subterms in $\operatorname{Right}\left(R_{E}\right)$ ) in the substitution part; second, by first normalizing the substitution w.r.t. $\curvearrowright_{R_{E}}$, which gives us an irreducible instance, we show that there are $B$-rewrite proofs for all instances.

This result, together with confluence of $\curvearrowright_{R_{E}}$, gives us that all consequences of $E$ have a $B$-rewrite proof using $R_{E}$.

Definition 16 (B-irreducible substitution). Let $E$ be a set of equations. $A$ substitution $\sigma$ is $B$-irreducible by $R_{E}$ in a term $t$ if, for all variables $x$ in $\operatorname{Dom}(\sigma)$, either

1. $x \sigma$ is irreducible by $R_{E}$ at every position in StrictContext $\left(x \sigma, \operatorname{Right}\left(R_{E}\right)\right)$, or

2. $x$ does not occur in $t$ at any position in StrictContext $\left(t \sigma, \operatorname{Right}\left(R_{E}\right)\right)$.

A substitution $\sigma$ is $B$-irreducible by $R_{E}$ in an equation $s \simeq t$ if $\sigma$ is $B$-irreducible by $R_{E}$ in both $s$ and $t$.

This irreducibility property is roughly inherited by the conclusion in all paramodulation inferences by $\mathcal{E}$ with rules in $R_{E}$.

Now we show that there is a $B$-rewrite proof for all instances of equations in $E$ with a $B$-irreducible substitution, provided that $E$ is closed under $\mathcal{E}$.

Lemma 29. Let $E$ be a set of equations that is closed under $\mathcal{E}, s \simeq t$ be an equation in $E$, and $\sigma$ be a substitution such that $(s \simeq t) \sigma$ is ground and $\sigma$ is

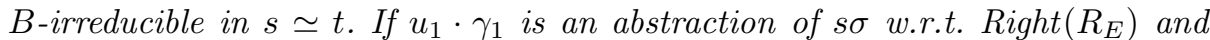
$u_{2} \cdot \gamma_{2}$ is an abstraction of t $\sigma$ w.r.t. Right $\left(R_{E}\right)$, then $u_{1} \cdot \gamma_{1} \curvearrowright_{R_{E}}^{*} u \cdot \gamma \curvearrowleft_{R_{E}}^{*} u_{2} \cdot \gamma_{2}$ for some abstracted term $u \cdot \gamma$.

Proof. We proceed by induction on size of the terms without counting the variables, i.e., $\left|u_{1}\right|_{v}+\left|u_{2}\right|_{v}$.

1. If either $u_{1} \gamma_{1}$ or $u_{2} \gamma_{2}$ have a term belonging to $\operatorname{Right}\left(R_{E}\right)$ at a non-variable position of $u_{1}$ or $u_{2}$ (respectively) then we abstract in this position. We show it for $u_{1}$ (for $u_{2}$ it is analogous). Let $p$ be a non-variable position of $u_{1}$ such that $\left.u_{1}\right|_{p} \gamma_{1} \in \operatorname{Right}\left(R_{E}\right)$. Then we have that $u_{1} \cdot \gamma_{1} \curvearrowright_{R_{E}} u_{1}[x]_{p} \cdot \gamma_{1}^{\prime}$, where $\gamma_{1}^{\prime}=\left(\gamma_{1} \cup\left\{\left.x \mapsto u_{1}\right|_{p} \gamma_{1}\right\}\right)$, and, by the induction hypothesis, it holds that $u_{1}[x]_{p} \cdot \gamma_{1}^{\prime} \curvearrowright_{R_{E}}^{*} u \cdot \gamma \curvearrowleft_{R_{E}}^{*} u_{2} \cdot \gamma_{2}$ for some abstracted term $u \cdot \gamma$. 
2. If neither $u_{1} \gamma_{1}$ nor $u_{2} \gamma_{2}$ have a term belonging to $\operatorname{Right}\left(R_{E}\right)$ at a nonvariable position of $u_{1}$ or $u_{2}$ (respectively), then there are two possibilities. If $u_{1} \cdot \gamma_{1} \equiv u_{2} \cdot \gamma_{2}$, then we are done. Otherwise, since $u_{1} \cdot \gamma_{1}$ is a maximal abstraction of $s \sigma$ (i.e., it cannot be further abstracted) and $u_{2} \cdot \gamma_{2}$ is a maximal abstraction of $t \sigma$, and they are not equivalent, we have that $s \sigma \not \equiv t \sigma$, and we can assume, w.l.o.g., that $s \sigma \succ t \sigma$.

Now, if $s \sigma \rightarrow t \sigma \in R_{E}$, then $t \sigma \in \operatorname{Right}\left(R_{E}\right)$ and, hence, $u_{2}$ is a variable $x$ and $\gamma_{2}$ is $\{x \mapsto t \sigma\}$, and $u_{1} \cdot \gamma_{1} \curvearrowright_{R_{E}} x \cdot\{x \mapsto t \sigma\}$. Therefore, taking $u=x$ and $\gamma=\gamma_{2}$, we are done.

If, otherwise, $s \sigma \rightarrow t \sigma \notin R_{E}$, then, by Definition 7 and Lemma 3, either $s \sigma$ or $t \sigma$ are reducible by $R_{E}$ at some position in $\operatorname{Strict} \operatorname{Context}\left(\sin \operatorname{Right}\left(R_{E}\right)\right)$ or in StrictContext $\left(t \sigma, \operatorname{Right}\left(R_{E}\right)\right)$, and hence at a non-variable position of $u_{1}$ or $u_{2}$ (respectively). We consider the case in which $s \sigma$ is reducible (the case for $t \sigma$ is analogous).

Let $p$ be a non-variable position of $u_{1}$ such that there is a rule $l \rightarrow r$ in $R_{E}$ with $\left.u_{1}\right|_{p} \gamma_{1} \equiv l$. Then we have that $u_{1} \cdot \gamma_{1} \curvearrowright_{R_{E}} u_{1}^{\prime} \cdot \gamma_{1}^{\prime}$, where $u_{1}^{\prime}=u_{1}[x]_{p}$ and $\gamma_{1}^{\prime}=\gamma_{1} \cup\{x \mapsto r\}$. On the other hand, since $\sigma$ is $B$-irreducible in $s \simeq t$, $p$ is a non-variable position of $s$ and, if $l^{\prime} \simeq r^{\prime}$ is an equation of $E$ such that, w.l.o.g., $l^{\prime} \sigma \equiv l$ and $r^{\prime} \sigma \equiv r$, then there is an inference by ordered paramodulation,

$$
\frac{l^{\prime} \simeq r^{\prime} \quad s \simeq t}{\left(s\left[r^{\prime}\right]_{p} \simeq t\right) \theta}
$$

where $\theta=\operatorname{mgu}\left(\left.s\right|_{p}, l^{\prime}\right)$, such that $\left(s\left[r^{\prime}\right]_{p} \simeq t\right) \theta \sigma$ is ground and $\sigma$ is $B$ irreducible in $\left(s\left[r^{\prime}\right]_{p} \simeq t\right) \theta$, since $r^{\prime} \theta \sigma \equiv r \in \operatorname{Right}\left(R_{E}\right)$ and $\sigma$ is $B$-irreducible in $s \simeq t$. Moreover, since $s\left[r^{\prime}\right]_{p} \theta \sigma \equiv s \sigma[r]_{p}$ and $t \theta \sigma \equiv t \sigma$, we have that $u_{1}^{\prime} \cdot \gamma_{1}^{\prime}$ is an abstraction of $s\left[r^{\prime}\right]_{p} \theta \sigma$ and $u_{2} \cdot \gamma_{2}$ is an abstraction of $t \theta \sigma$. Finally, either $u_{1}^{\prime} \gamma_{1}^{\prime} \equiv u_{2} \gamma_{2}$ and hence we have that $u_{1} \cdot \gamma_{1} \curvearrowright_{R_{E}}^{*} u \cdot \gamma \curvearrowleft_{R_{E}}^{*} u_{2} \cdot \gamma_{2}$ for some abstracted term $u \cdot \gamma$ by abstraction steps, or else the conclusion of the inference is not a tautology and hence is in $E$ and, since $\sigma$ is $B$-irreducible in the conclusion and $\left|u_{1}\right|_{v}>\left|u_{1}^{\prime}\right|_{v}$ then, by the induction hypothesis, we have that $u_{1}^{\prime} \cdot \gamma_{1}^{\prime} \curvearrowright_{R_{E}}^{*} u \cdot \gamma \curvearrowleft_{R_{E}}^{*} u_{2} \cdot \gamma_{2}$ for some abstracted term $u \cdot \gamma$.

Now, as said before, we show that we can obtain a $B$-rewrite proof using $R_{E}$ for every instance of an equation in $E$, by first normalizing the substitution w.r.t. $\curvearrowright_{R_{E}}$.

Lemma 30. Let $E$ be a set of equations that is closed under $\mathcal{E}$, and $s \simeq t$ be an equation in $\operatorname{gnd}(E)$. Then $s \curvearrowright_{R_{E}}^{*} u \cdot \gamma \curvearrowleft_{R_{E}}^{*} t$ for some abstracted term $u \cdot \gamma$.

Proof. Let $s \simeq t$ be an instance of an equation $s^{\prime} \simeq t^{\prime}$ in $E$, such that $s \equiv s^{\prime} \sigma$ and $t \equiv t^{\prime} \sigma$ for some substitution $\sigma$. First of all we normalize $\sigma$, i.e., we define a new substitution $\sigma^{\prime}$ such that $x \sigma^{\prime} \equiv w \gamma_{w}$ if $x \sigma{\stackrel{!}{R_{E}}} w \cdot \gamma_{w}$. Then there is an equation in $\operatorname{gnd}(E)$ of the form $s^{\prime} \sigma^{\prime} \simeq t^{\prime} \sigma^{\prime}$, where $\sigma^{\prime}$ is $B$-irreducible in $s^{\prime} \simeq t^{\prime}$. Moreover, we have that $s \curvearrowright_{R_{E}}^{*} u_{1} \cdot \gamma_{1}$ and $t \curvearrowright_{R_{E}}^{*} u_{2} \cdot \gamma_{2}$ for some abstractions $u_{1} \cdot \gamma_{1}$ and $u_{2} \cdot \gamma_{2}$ of $s^{\prime} \sigma^{\prime}$ and $t^{\prime} \sigma^{\prime}$ w.r.t. $\operatorname{Right}\left(R_{E}\right)$, respectively (these abstracted terms are 
easily obtained from the normalization of the substitution, i.e., by abstracting each $\left.w \gamma_{w}\right)$. Finally, by Lemma 29, we have that $u_{1} \cdot \gamma_{1} \curvearrowright_{R_{E}}^{*} u \cdot \gamma \curvearrowleft_{R_{E}}^{*} u_{2} \cdot \gamma_{2}$, which allows us to conclude by composing the rewriting steps.

The following theorem follows directly from the previous lemma. Note that rewriting with $\curvearrowright_{R_{E}}$ also provides a rewrite sequence with $R_{E}$.

Theorem 3. Let $E$ be a set of equations that is closed under $\mathcal{E}$. Then $R_{E}^{*} \models E$.

Now, together with confluence of $\curvearrowright_{R_{E}}$, we can show that all consequences of $E$ have a $B$-rewrite proof.

Lemma 31. Let $E$ be a set of equations that is closed under $\mathcal{E}$. If $E \models s \simeq t$ for some ground terms $s$ and $t$, then $s \curvearrowright_{R_{E}}^{*} u \cdot \gamma \curvearrowleft_{R_{E}}^{*} t$ for some abstracted term $u \cdot \gamma$.

Proof. Since $E \models s \simeq t$, we can go from $s$ to $t$ using the equations in $\operatorname{gnd}(E)$. Then there is a sequence of terms $s_{0}, s_{1} \ldots, s_{n}$ such that $s_{0}=s$ and $s_{n}=t$ and, for every $i$ in $\{0, \ldots, n-1\}$, we have that $s_{i}=C\left[e_{i}\right]$ and $s_{i+1}=C\left[e_{i+1}\right]$ for some context $C$ and equation $e_{i} \simeq e_{i+1}$ in $\operatorname{gnd}(E)$. By Lemma 30 and monotonicity of $\curvearrowright_{R_{E}}$, for every $i$ in $\{0, \ldots, n-1\}$, we have that $s_{i} \curvearrowright_{R_{E}}^{*} u_{i} \cdot \gamma_{i} \curvearrowleft_{R_{E}}^{*} s_{i+1}$ for some abstracted term $u_{i} \cdot \gamma_{i}$. Finally, by confluence of $\curvearrowright_{R_{E}}$, we have that $s=s_{0} \curvearrowright_{R_{E}}^{*} u \cdot \gamma \curvearrowleft_{R_{E}}^{*} s_{n+1}=t$ for some abstracted term $u \cdot \gamma$.

This result implies that we have obtained a Knuth-Bendix completion procedure for ordered rewriting with well-founded orderings.

\section{The Horn case}

In this section we generalize the results of the previous section to Horn clauses. In the following inference system it is assumed that, in each clause with a non-empty antecedent, one of the negative equations, the one that is written underlined, has been selected.

Definition 17. The inference system $\mathcal{H}$ for Horn clauses with respect to a wellfounded ordering $\succ$ is defined as follows:

paramodulation right:

$$
\begin{aligned}
& \frac{\rightarrow l \simeq r \quad \rightarrow s \simeq t}{\rightarrow\left(s[r]_{p} \simeq t\right) \sigma} \quad \text { where } \sigma=\operatorname{mgu}\left(l,\left.s\right|_{p}\right) \\
& \text { paramodulation left: } \\
& \frac{\rightarrow l \simeq r \quad \Gamma, \underline{s \simeq t \rightarrow \Delta}}{\left(\Gamma, s[r]_{p} \simeq t \rightarrow \Delta\right) \sigma} \quad \text { where } \sigma=\operatorname{mgu}\left(l,\left.s\right|_{p}\right) \\
& \text { equality resolution: }
\end{aligned}
$$

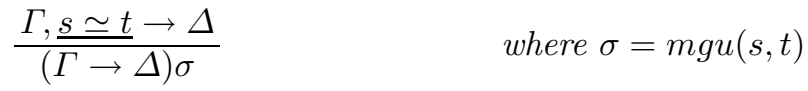


where, moreover, in both paramodulation rules $l$ is maximal in its premise (that is, for some ground substitution $\theta$, it holds that $l \sigma \theta \succ r \sigma \theta)$, and $\left.s\right|_{p}$ is not a variable except in the paramodulation right inference when $s$ and $l$ are both variables. See Definition 3 for further details on the application of this inference rule.

Definition 18. Let $S$ be a set of Horn clauses. By $E_{S}$ we denote the set of equations occurring as positive unit clauses in $S$, i.e., $E_{S}=\{s \simeq t \mid \rightarrow s \simeq t \in$ $S\}$, and by $R_{S}$ we denote the set of rules generated by $E_{S}$ as in Definition $\%$.

First we show that, if $S$ is a set of clauses that is closed under $\mathcal{H}$ and does not contain the empty clause, then $R_{S}^{*}$ is a model for all clauses in $S$ that are $B$-irreducible by $R_{S}$.

A substitution $\sigma$ is $B$-irreducible by $R_{S}$ in a clause $\Gamma \rightarrow \Delta$ if $\sigma$ is $B$ irreducible by $R_{S}$ in all equations $s \simeq t$ (see Definition 16) occurring in $\Gamma$ or $\Delta$.

Lemma 32. Let $S$ be a set of Horn clauses that is closed under $\mathcal{H}$, such that $\square \notin S$. Then $R_{S}^{*} \models C \sigma$ for all clauses $C$ in $S$ and ground substitutions $\sigma$ that are $B$-irreducible by $R_{S}$ in $C$.

Proof. Given a clause $C$, by $|C|_{v}$ we denote the number of equations in $C$, plus the sum of the size of the terms occurring in $C$ as a side of an equation, without counting the variables.

We proceed by minimal counterexample. Let $C^{\prime} \cdot \gamma$ be the abstracted clause w.r.t. $\operatorname{Right}\left(R_{S}\right)$ with minimal $\left|C^{\prime}\right|_{v}$ such that $C^{\prime} \cdot \gamma$ is an abstraction of a clause $C \sigma$ with $C \in S, \sigma B$-irreducible by $R_{S}$ in $C$, and $R_{S}^{*} \not \models C \sigma$.

Since $E_{S}$ is closed under ordered paramodulation then, by Theorem 3, we have that $R_{S}^{*} \models E_{S}$ and, therefore, $C \sigma$ cannot be a positive unit clause. Then, since $\square \notin S, C$ is of the form $\Gamma, \underline{s \simeq t} \rightarrow \Delta$ and $C^{\prime}$ is of the form $\Gamma^{\prime}, \underline{s^{\prime} \simeq t^{\prime}} \rightarrow \Delta^{\prime}$. Two cases have to be considered:

1. If $s \sigma \equiv t \sigma$, then there is an inference by equality resolution,

$$
\frac{\Gamma, \underline{s \simeq t} \rightarrow \Delta}{(\Gamma \rightarrow \Delta) \theta},
$$

whose conclusion $D$ has an instance $D \sigma$ such that $\left(\Gamma^{\prime} \rightarrow \Delta^{\prime}\right) \cdot \gamma$ is an abstraction of $D \sigma$ with $\left|C^{\prime}\right|_{v}>\left|\Gamma^{\prime} \rightarrow \Delta^{\prime}\right|_{v}$ and, moreover, $\sigma$ is $B$-irreducible by $R_{S}$ in $D$ and $R_{S}^{*} \not \models D \sigma$, which is a contradiction.

2. If $s \sigma \not \equiv t \sigma$, since $R_{S}^{*} \not \models C \sigma$, we have that $R_{S}^{*} \models s \sigma \simeq t \sigma$. Then, since $R_{S} \subseteq E_{S}$, by Lemma 31 there must be a $B$-rewrite proof of $s \sigma \simeq t \sigma$ with $R_{S}$. Therefore, since $s \sigma \curvearrowright_{R_{S}}^{*} s^{\prime} \cdot \gamma$ and $t \sigma \curvearrowright_{R_{S}}^{*} t^{\prime} \cdot \gamma$ (using abstraction steps), by confluence of $\curvearrowright_{R_{S}}$, there is some abstracted term $u \cdot \delta$ such that $s^{\prime} \cdot \gamma \curvearrowright_{R_{S}}^{*} u \cdot \delta \curvearrowleft_{R_{S}}^{*} t^{\prime} \cdot \gamma$. Then, since $s^{\prime} \gamma \not \equiv t^{\prime} \gamma$, either $s^{\prime} \cdot \gamma$ or $t^{\prime} \cdot \gamma$ is reducible by $\curvearrowright_{R_{S}}$ with a non-abstraction step. Assume that $s^{\prime} \cdot \gamma$ is reducible (the other case is analogous) at some non-variable position $p$ of $s^{\prime}$. By $B$-irreducibility 
of the substitution $\sigma$ in $C$ and minimality of $\left|C^{\prime}\right|_{v}, p$ must be a non-variable position of $s$ and, hence, there is an inference by paramodulation left,

$$
\frac{\rightarrow l \simeq r \quad \Gamma, \underline{s \simeq t \rightarrow \Delta}}{\left(\Gamma, s[r]_{p} \simeq t \rightarrow \Delta\right) \theta},
$$

whose conclusion $D$ has an instance $D \sigma$ such that $\left(\Gamma^{\prime}, s^{\prime}[x]_{p} \simeq t^{\prime} \rightarrow \Delta^{\prime}\right)$. $(\gamma \cup\{x \mapsto r \sigma\})$ is an abstraction of $D \sigma$ with $\left|C^{\prime}\right|_{v}>\left|\Gamma^{\prime}, s^{\prime}[x]_{p} \simeq t^{\prime} \rightarrow \Delta^{\prime}\right|_{v}$ and, moreover, $\sigma$ is $B$-irreducible by $R_{S}$ in $D$ (note that $r \sigma$ is a term in $\left.\operatorname{Right}\left(R_{E}\right)\right)$ and $R_{S}^{*} \not \forall D \sigma$, which is a contradiction.

Now, as in the equational case, by first normalizing the substitution, we can prove the main theorem.

Theorem 4 (Refutation completeness of $\mathcal{H}$ for Horn clauses). Let $S$ be a set of Horn clauses that is closed under $\mathcal{H}$. Then $\square \in S$ if, and only if, $S$ is unsatisfiable.

Proof. The left-to-right implication is trivial. For the other one, let $S$ be a set of clauses closed under $\mathcal{H}$ such that $\square \notin S$. We prove that $R_{S}^{*}$ is a model of $S$, i.e., that $R_{S}^{*} \models C \sigma$ for all clauses $C$ in $S$ and ground substitutions $\sigma$. Let $\sigma^{\prime}$ be a substitution such that $x \sigma^{\prime} \equiv w \gamma_{w}$ if $x \sigma \curvearrowright_{R_{S}}^{\vdots} w \cdot \gamma_{w}$. Then $R_{S} \cup\left\{C \sigma^{\prime}\right\} \models C \sigma$ and, since $\sigma^{\prime}$ is $B$-irreducible by $R_{S}$ in $C$, by Lemma 32, we have that $R_{S}^{*} \models C \sigma^{\prime}$, which implies that $R_{S}^{*} \models C \sigma$.

\section{Conclusion}

In this paper we have improved on the result of [7], by showing that wellfoundedness of the term ordering is sufficient for completeness of (positive) ordered paramodulation for Horn clauses with equality. In [7], orderings need not be monotone but have to be well-founded and include the subterm relation. In our result the latter requirement has been dropped, and thus equations can even be ordered from subterms to superterms as in $a \rightarrow f(a)$, in an unfolding way.

This is a non-trivial extension of the previous one, since rules of the form $a \rightarrow f(a)$ necessarily cause non-termination of standard rewriting. In the completeness proof we show the confluence of a non-terminating limit rewrite system where lefthand sides may overlap, and which is applied using a restricted and terminating form of rewriting, called blocked rewriting (B-rewriting for short). Interestingly, a by-product of our results is a Knuth-Bendix completion procedure for ordered rewriting with well-founded orderings, and its completeness. We have pointed out two potential applications of our result:

- The first one is in reference to deduction modulo built-in equational theories $E$, since there is always a well-founded $E$-compatible ordering and, hence, requiring only well-foundedness allows us to consider any theory $E$. We hope that the shorter and easier to read proof that we have presented here can open the door to extend our completeness results to deduction modulo any theory $E$ with a decidable $E$-unification problem. 
- The second one concerns goal-oriented proofs. It turns out that, sometimes, a goal-oriented (ordered) paramodulation proof can only be obtained if the ordering contradicts the subterm property (see Example 2 of Section 1).

Although these potential applications are interesting, additional techniques for redundancy elimination, like simplification by rewriting, should be investigated in this framework to make the calculus useful in practice. Note that from our proof only tautology deletion is clearly possible. Subsumption is difficult to fit in our approach to the completeness of the calculus, since it greatly interferes with the proof of the so-called Return Property, which is a cornerstone of our completeness result. However, we have found no counterexample showing that subsumption can cause incompleteness. Moreover, like in [7], similar problems concerning the use of demodulation, i.e., simplification by rewriting, arise. As a hint, note that, in order to fulfill the Return Property, one may need to include pairs of rules like

$$
\begin{aligned}
a & \rightarrow f(a) \\
f(f(a)) & \rightarrow f(a)
\end{aligned}
$$

which is not compatible with demodulation, since the second one would be removed.

The problem of providing concrete redundancy notions for paramodulation with non-monotonic orderings has been addressed in [9], where results on restricted forms of demodulation for paramodulation with well-founded orderings fulfilling the subterm property, are given. There, some examples of incompleteness when full demodulation is applied are provided. These examples, obviously also apply if only well-foundedness is required.

$B$-rewriting is closely related to basic strategies (see $[5,15]$ ), which we believe can be compatible with our results. The basicness results should be worked out using the notion of closures defined for clauses in [5], since it coincides, at the level of clauses, with our abstracted terms (which we have used only in the proofs).

The extension of our results to full first-order clauses is not easy. The problem is that, within the model generation proof technique, the completeness for general clauses is usually based on the fact that, at most, one positive equation is satisfied in the generated (rewrite) model. Since, in our case, the ordering can contradict the subterm relation, we do not know how to obtain such a minimal model. The source of the problem is that the satisfiability of some equation in a clause $C$, may follow from rules that are added to the model later than when $C$ was considered. Let us illustrate this situation with a simple example:

Example 8. Let $\succ$ be a (total) well-founded ordering on ground terms such that

$$
g(f(a)) \succ b \succ g(a) \succ f(f(a)) \succ a \succ f(a)
$$


and $E$ be a set of equations that is closed under ordered paramodulation, containing

$$
\begin{aligned}
(E 1) \quad a & \simeq f(a), \\
(E 2) \quad f(f(a)) & \simeq f(a), \\
(E 3) \quad b & \simeq g(a), \\
(E 4) \quad g(f(a)) & \simeq g(a), \quad \text { and } \\
(E 5) \quad g(f(a)) & \simeq b
\end{aligned}
$$

among other (greater) equations. Then the generated $R_{E}$ would be

$$
\begin{array}{lll}
(R 1) & a & \rightarrow f(a) \\
(R 2) & f(f(a)) & \rightarrow f(a) \\
(R 3) & g(f(a)) & \rightarrow b,
\end{array}
$$

since both $b \rightarrow g(a)$ and $g(f(a)) \rightarrow g(a)$ are reducible by $a \rightarrow f(a)$ at some position of their righthand side which is not below any previously known righthand side and, hence, they do not generate a rule in $R_{E}$. However, $b \simeq g(a)$ is finally fulfilled by the model, since it follows from $a \simeq f(a)$ and $g(f(a)) \simeq b$, where the latter is a greater equation.

In the case of full first-order clauses, this could imply that a non-unit positive clause which has generated a rule in $R_{E}$ because none of its equations was satisfied at that moment, has at the end more than one equation satisfied by the final model, which breaks the standard completeness proof.

In spite of the limitations of the presented calculus, we think that this is a new important step in the theory of paramodulation, which shows the power of ordered paramodulation regardless of the properties of the ordering that is used, and leaves as the last question whether even well-foundedness is necessary.

\section{$7 \quad$ Funding}

This work was supported by the Spanish Ministry of Science and Technology through the LogicTools-2 (TIN2007-68093-C02-01) to A.R.

\section{References}

1. L. Bachmair and N. Dershowitz. Equational inference, canonical proofs, and proof orderings. Journal of the ACM, 41(2):236-276, Feb. 1994.

2. L. Bachmair, N. Dershowitz, and J. Hsiang. Orderings for equational proofs. In First IEEE Symposium on Logic in Computer Science (LICS), pages 346-357, Cambridge, MA, USA, June 16-18, 1986. IEEE Computer Society Press, Los Alamitos, CA, USA.

3. L. Bachmair and H. Ganzinger. Rewrite-based equational theorem proving with selection and simplification. Journal of Logic and Computation, 4(3):217-247, 1994. 
4. L. Bachmair and H. Ganzinger. Equational reasoning in saturation-based theorem proving. In W. Bibel and P. Schmitt, editors, Automated Deduction - A Basis for Applications, volume I, chapter 11, pages 353-397. Kluwer, Dordrecht, The Netherlands, 1998.

5. L. Bachmair, H. Ganzinger, C. Lynch, and W. Snyder. Basic paramodulation. Information and Computation, 121(2):172-192, 1995.

6. M. Bofill, G. Godoy, R. Nieuwenhuis, and A. Rubio. Paramodulation with nonmonotonic orderings. In 14th IEEE Symposium on Logic in Computer Science (LICS), pages 225-233, Trento, Italy, July 2-5, 1999. IEEE Computer Society Press, Los Alamitos, CA, USA.

7. M. Bofill, G. Godoy, R. Nieuwenhuis, and A. Rubio. Paramodulation and KnuthBendix Completion with Nontotal and Nonmonotonic Orderings. Journal of Automated Reasoning, 30(1):99-120, 2003.

8. M. Bofill and A. Rubio. Well-foundedness is sufficient for completeness of ordered paramodulation. In 18th International Conference on Automated Deduction (CADE), volume 2392 of LNAI, pages 456-470, Copenhagen, Denmark, July 27-30 2002. Springer-Verlag, Berlin Heidelberg, Germany.

9. M. Bofill and A. Rubio. Redundancy notions for paramodulation with nonmonotonic orderings. In 2nd International Joint Conference on Automated Reasoning (IJCAR), volume 3097 of LNAI, pages 107-121, Cork, Ireland, July 4-8 2004. Springer-Verlag, Berlin Heidelberg, Germany.

10. N. Dershowitz and J.-P. Jouannaud. Rewrite systems. In J. van Leeuwen, editor, Handbook of Theoretical Computer Science, volume B: Formal Models and Semantics, chapter 6, pages 244-320. Elsevier Science B.V, Amsterdan, The Netherlands and The MIT Press, Cambridge, MA, USA, 1990.

11. J. Hsiang and M. Rusinowitch. Proving refutational completeness of theorem proving strategies: the transfinite semantic tree method. Journal of the ACM, 38(3):559-587, jul 1991.

12. C. Marché. On ground AC-completion. In 4th International Conference on Rewriting Techniques and Applications (RTA), number 488 in LNCS, pages 411-422, Como, Italy, Apr. 10-12, 1991. Springer-Verlag, Berlin Heidelberg, Germany.

13. P. Narendran and M. Rusinowitch. Any ground associative commutative theory has a finite canonical system. In 4th Int. Conf. Rewriting Techniques and Applications (RTA), number 488 in LNCS, pages 423-434, Como, Italy, Apr. 10-12, 1991. Springer-Verlag, Berlin Heidelberg, Germany.

14. P. Narendran and M. Rusinowitch. The Unifiability Problem in Ground AC Theories. In 8th Annual IEEE Symposium on Logic in Computer Science (LICS), pages 364-370, Montreal, Canada, June 19-23, 1993. IEEE Computer Society Press, Los Alamitos, CA, USA.

15. R. Nieuwenhuis and A. Rubio. Theorem Proving with Ordering and Equality Constrained Clauses. Journal of Symbolic Computation, 19(4):321-351, April 1995.

16. R. Nieuwenhuis and A. Rubio. Paramodulation with Built-in AC-Theories and Symbolic Constraints. Journal of Symbolic Computation, 23(1):1-21, May 1997.

17. R. Nieuwenhuis and A. Rubio. Paramodulation-based theorem proving. In J. Robinson and A. Voronkov, editors, Handbook of Automated Reasoning, volume 1, chapter 7, pages 372-444. Elsevier Science B.V, Amsterdan, The Netherlands and The MIT Press, Cambridge, MA, USA, 2001.

18. A. Rubio and R. Nieuwenhuis. A total AC-compatible ordering based on RPO. Theoretical Computer Science, 142(2):209-227, May 15, 1995. 
19. M. Rusinowitch and L. Vigneron. Automated deduction with associative commutative operators. Journal of Applicable Algebra in Engineering, Communication and Computation, 6(1):23-56, 1995.

20. W. Wechler. Universal Algebra for Computer Scientists, volume 25 of EATCS Monographs on Theoretical Computer Science. Springer-Verlag, Berlin Heidelberg, Germany, 1991. 